\title{
The soft $x$-ray properties of a complete sample of optically selected quasars. 1: First results
}

\section{Citation}

Laor, Ari, Fabrizio Fiore, Martin Elvis, Belinda J. Wilkes, and Jonathan C. McDowell. 1994. "The Soft x-Ray Properties of a Complete Sample of Optically Selected Quasars. 1: First Results." The Astrophysical Journal 435 (November): 611. doi:10.1086/174841.

\section{Published Version}

doi:10.1086/174841

\section{Permanent link}

http://nrs.harvard.edu/urn-3:HUL.InstRepos:30212137

\section{Terms of Use}

This article was downloaded from Harvard University's DASH repository, and is made available under the terms and conditions applicable to Other Posted Material, as set forth at http:// nrs.harvard.edu/urn-3:HUL.InstRepos:dash.current.terms-of-use\#LAA

\section{Share Your Story}

The Harvard community has made this article openly available.

Please share how this access benefits you. Submit a story.

Accessibility 
THE SOFT X-RAY PROPERTIES OF A COMPLETE SAMPLE OF OPTICALLY SELECTED QUASARS. I. FIRST RESULTS

\author{
Ari LaOr, ${ }^{1}$ Fabrizio Fiore, ${ }^{2,3}$ Martin Elvis, ${ }^{2}$ Belinda J. Wilkes, ${ }^{2}$ AND Jonathan C. MCDowell ${ }^{2}$ \\ Received 1993 October 21; accepted 1994 May 11
}

\begin{abstract}
We present the results of ROSAT PSPC observations of 10 quasars. These objects are part of our ROSAT program to observe a complete sample of optically selected quasars. This sample includes all 23 quasars from the bright quasar survey with a redshift $z \leq 0.400$ and a Galactic $\mathrm{H}_{\text {I }}$ column density $N_{\mathrm{HI}}^{\mathrm{Gal}}<1.9 \times 10^{20} \mathrm{~cm}^{-2}$. These selection criteria, combined with the high sensitivity and improved energy resolution of the PSPC, allow us to determine the soft $(\sim 0.2-2 \mathrm{keV}) \mathrm{X}$-ray spectra of quasars with about an order of magnitude higher precision compared with earlier soft X-ray observations.

The following main results are obtained: Strong correlations are suggested between the soft X-ray spectral slope $\alpha_{x}$ and the following emission line parameters: $\mathrm{H} \beta$ FWHM, $L_{[\mathrm{O} \text { II] }}$, and the Fe II/H $\beta$ flux ratio. These correlations imply the following: (1) The quasar's environment is likely to be optically thin down to $\sim 0.2$ keV. (2) In most objects $\alpha_{x}$ varies by less than $\sim 10 \%$ on timescales shorter than a few years. (3) $\alpha_{x}$ might be a useful absolute luminosity indicator in quasars. (4) The Galactic He I and H I column densities are well correlated.

Most spectra are well characterized by a simple power law, with no evidence for either significant absorption excess or emission excess at low energies, to within $\sim 30 \%$. We find $\left\langle\alpha_{x}\right\rangle=-1.50 \pm 0.40$, which is consistent with other ROSAT observations of quasars. However, this average is significantly steeper than suggested by earlier soft X-ray observations of the Einstein IPC. The $0.3 \mathrm{keV}$ flux in our sample can be predicted to better than a factor of 2 once the $1.69 \mu \mathrm{m}$ flux is given. This implies that the X-ray variability power spectra of quasars flattens out between $f \sim 10^{-5}$ and $f \sim 10^{-8} \mathrm{~Hz}$.

A steep $\alpha_{x}$ is mostly associated with a weak hard X-ray component, relative to the near-IR and optical emission, rather than a strong soft excess, and the scatter in the normalized $0.3 \mathrm{keV}$ flux is significantly smaller than the scatter in the normalized $2 \mathrm{keV}$ flux. This argues against either thin or thick accretion disks as the origin of the soft X-ray emission. Further possible implications of the results found here are briefly discussed.
\end{abstract}

Subject headings: accretion, accretion disks - ISM : abundances - quasars: general — X-rays: galaxies

\section{INTRODUCTION}

Despite 30 years of intensive research the energy source of quasars is not yet established. Quasars have been detected over a very wide energy range, from the radio to hard $\gamma$-rays. However, more than half of their extreme power is typically concentrated over a relatively small energy range, from the optical to soft X-rays (e.g., Sanders et al. 1989). The UV power generally rises with increasing energy, while the soft X-ray power (below $\sim 1 \mathrm{keV}$ ) generally falls with increasing energy, indicating that the peak power of quasars is emitted somewhere in the extreme UV (a few hundred angstroms). An accurate determination of the continuum emission of quasars near their power peak energy is important for testing and constraining possible emission mechanisms.

The large Galactic opacity prevents a direct observation of the extreme UV in quasars. One alternative is to observe the UV spectra of very high redshift quasars (e.g., Reimers et al. 1992); however, this approach is limited by the high abundance

\footnotetext{
${ }^{1}$ School of Natural Sciences, Institute for Advanced Study; Current address: Theoretical Astrophysics, Caltech 130-33, Pasadena, CA 91125; laor@tapir.caltech.edu.

2 Harvard-Smithsonian Center for Astrophysics, 60 Garden Street, Cambridge, MA 02138; elvis@cfa222.harvard.edu, belinda@cfa222.harvard.edu, mcdowell@urania.harvard.edu.

${ }^{3}$ Current address Osservatorio Astronomico di Roma, via dell'Osservatorio 5, Monteporzio-Catone (RM), 00040 Italy; fiore\%astrmp.decnet@cfa.harvard.edu.
}

of Lyman limit systems at $z \gtrsim 3$. The other alternative, adopted here, is to go to the other side of the Galactic opacity barrier and to observe low-redshift quasars in very soft $X$-rays.

The X-ray properties of quasars have been studied extensively over the last decade using $H E A O-1$, Einstein, EXOSAT, and Ginga (e.g., Mushotzky 1984; Wilkes \& Elvis 1987; Canizares \& White 1989; Comastri et al. 1992; Lawson et al. 1992; Williams et al. 1992; and a recent review by Mushotzky, Done, \& Pounds 1993). These observations indicate that the X-ray emission above $1-2 \mathrm{keV}$ is well described by a power law with a spectral slope $\alpha_{x}=d \ln f_{v} / d \ln v$ of about -0.5 for radio-loud quasars and about -1.0 for radio-quiet quasars.

As mentioned above, X-ray observations below $1 \mathrm{keV}$ indicate a spectral steepening, or equivalently an excess emission, relative to the flux predicted by an extrapolation of the hard X-ray power law (e.g., Arnaud et al. 1985; Wilkes \& Elvis 1987; Turner \& Pounds 1989; Masnou et al. 1992; Comastri et al. 1992). In some objects the excess can be described as a very steep and soft component, which is consistent with the Wien tail of a hot thermal component dominating the UV emission. However, these studies were limited by the low signal-to-noise ratio $(\mathbf{S} / \mathbf{N})$ and energy resolution of the Einstein IPC and the EXOSAT LE detectors, in particular in the crucial energy range below $0.5 \mathrm{keV}$. This prevented an accurate determination of the soft X-ray emission spectrum of quasars. Furthermore, the objects studied do not form a complete sample, and these results are likely to be biased by various selection effects 
which were not well defined a priori. In particular, most studied objects are nearby, intrinsically X-ray bright, active galactic nuclei (AGNs).

The PSPC detector aboard ROSAT has a significantly improved sensitivity, energy resolution $(E / \Delta E=2.4(E / 1$ $\mathrm{keV})^{1 / 2} \mathrm{FWHM}$ ), and spatial resolution below $\sim 2 \mathrm{keV}$, compared with previous detectors (Trümper 1983). We use this detector to make an accurate determination of the soft X-ray properties of a well-defined, complete, and otherwise wellexplored, sample of quasars.

This survey will allow us to address the following questions: (1) What are the soft X-ray spectral properties of typical, optically selected, low-redshift quasars? (2) Are simple thin accretion disk models (e.g., Laor 1990) able to fit the observed optical/UV/soft X-ray continuum? Are other modifying mechanisms, such as a hot corona (e.g., Czerny \& Elvis 1987) required? Are models invoking optically thin free-free emission (e.g., Ferland, Korista, \& Peterson 1990; Barvainis 1993) possible? (3) Do the observed soft X-ray properties display any significant correlations with other properties of these quasars? Are these correlations compatible with various models for the continuum and line emission mechanisms?

In this paper we report the results of the analysis of the ROSAT PSPC spectra of 10 quasars already available to us. These spectra suggest a number of strong and surprising correlations, whose possible physical implications are briefly discussed. The outline of the paper is as follows. In $\S 2$ we describe the sample selection criteria; in $\S 3$ we describe the observations and the analysis of the spectra. Section 4 describes the analysis of correlations between the soft X-ray properties and other continuum and emission line properties. In $\S 5$ we compare our results with other soft X-ray observations and discuss some of the implications. We conclude in $\S 6$ with a summary of our main results.

\section{THE SAMPLE}

We found the bright quasar survey (BQS) sample, a subset of the PG survey defined by Schmidt \& Green (1983), to be particularly suitable for our purpose for the following reasons:

1. These objects are selected only by their optical properties, thus they are not biased in any direct way in terms of their $\mathrm{X}$-ray properties.

2. This sample has already been studied extensively and in a uniform manner in other parts of the spectrum including the radio by Kellerman et al. (1989) and Miller, Rawlings, \& Saunders (1993), the mid- to far-IR by Sanders et al. (1989), the near-IR to optical by Neugebauer et al. (1987), and the 0.3-3.5 keV X-rays by Tananbaum et al. (1986; see also Della Ceca et al. 1990); optical spectroscopy is reported in Baldwin, Wampler, \& Gaskell (1989), and Boroson \& Green (1992); IUE spectra of a large fraction $(71 \%)$ of the $P G$ quasars are given in Lanzetta, Turnshek, \& Sandoval (1993); the optical polarization was studied by Berriman et al. (1990). These studies of the PG quasars provide us with the most complete and coherent picture of the emission properties of bright AGNs, and allow us to make a detailed study of possible correlations between the soft X-ray properties and various other emission properties.

3. This sample includes a large fraction of the brightest known quasars, thus high-S/N spectra could be obtained within a reasonable amount of spacecraft time.

The complete PG sample includes 114 AGNs, of which 92 are quasars (i.e., $M_{B}<-23$ ). These objects were selected by the color criterion $U-B<-0.44$, which according to Schmidt \& Green is satisfied by $88 \%$ of the quasars listed in the Hewitt \& Burbidge (1980) catalog. Thus, the PG quasars are likely to be representative of most quasars. We select a subsample of the PG quasars which is optimally suitable for soft X-ray observations. The following two selection criteria were used: (1) $z \leq 0.400$. This prevents the rest frame $0.2 \mathrm{keV}$ from being redshifted beyond the observable range. (2) $N_{\mathrm{HI}}^{\mathrm{Gal}}<$ $1.9 \times 10^{20} \mathrm{~cm}^{-2}$, where $N_{\mathrm{HI}}^{\mathrm{Gal}}$ is the $\mathrm{H}$ I Galactic column density as measured in $21 \mathrm{~cm}$. This low $N_{\mathrm{HI}}^{\mathrm{Gal}}$ cutoff is critical for minimizing the effects of Galactic absorption. This cutoff implies an upper limit on the Galactic optical depth in our sample of $\tau_{0.2 \mathrm{kev}}<1.6$ (Morrison \& McCammon 1983). However, even with the low $N_{\mathrm{HI}}^{\mathrm{Gal}}$ cutoff, no photons below 0.15 $\mathrm{keV}$ can be detected. This is because the opacity of the Galaxy increases as $\sim E^{-3}$, giving $\tau_{0.1 \mathrm{keV}}=N_{\mathrm{H}} / 1.77 \times 10^{19}$, while the effective area of the PSPC drops rapidly below $0.15 \mathrm{keV}$. As a result practically no photons below $0.15 \mathrm{keV}$ can be detected from the quasars (although the formal lower limit of the usable channels on the PSPC is $0.1 \mathrm{keV}$ ). These criteria limit our sample to 23 quasars, which we expect to be representative of the low-redshift, optically selected quasar population.

The complete sample of 23 quasars is listed in Table 1 together with their redshifts, $m_{B}$ and $M_{B}$ magnitudes (calculated for $H_{0}=50 \mathrm{~km} \mathrm{~s}^{-1}, q=0.5$ ), $R$ the radio-tooptical flux ratio, and $N_{\mathrm{HI}}^{\mathrm{Gal}}$. The redshifts and $m_{B}$ and $M_{B}$ magnitudes are taken from Schmidt \& Green (1983), $R$ is taken from Kellerman et al. (1989), and the $N_{\mathrm{HI}}^{\mathrm{Gal}}$ are taken from the large-beam $N_{\mathrm{HI}}^{\mathrm{Gal}}$ survey by Stark et al. (1992), or when available, from the small-beam accurate measurement by Elvis, Lockman, \& Wilkes (1989) or Savage et al. (1993). The average $N_{\mathrm{HI}}^{\mathrm{Gal}}$ in our sample is $1.52 \times 10^{20} \mathrm{~cm}^{-2}$, which is about half the typical value at high Galactic latitudes (Dickey \& Lockman 1990).

Note that four of the 23 quasars in our sample are radioloud (defined here as $R \geq 10$ ), which is comparable to the ratio of $22 \%(20 / 92)$ in the complete PG sample (Kellerman et al.). Einstein IPC observations are available for eight of the quasars in our sample (Wilkes et al. 1994). These observations will be discussed in a future analysis of the soft X-ray variability properties of our objects. The 10 quasars reported here were selected from the complete sample of 23 quasars on the basis of their availability, and we do not expect a systematic bias in their intrinsic properties compared with the complete sample.

\section{THE OBSERVATIONS AND ANALYSIS OF THE SPECTRA}

Table 2 lists the observations of the 10 quasars analyzed here. One quasar of the 10 (3C 273) was obtained from the ROSAT public archives. All the quasars were readily detected with a minimum of $\sim 600$ net counts, and typically $\sim 2000$. We used the PROS software package to extract the source counts. Table 2 includes the exposure times, the dates of the observations, the net number of counts and their statistical error, the count rate, the radius of the circular aperture used to extract the source counts, the offset of the X-ray position from the optical position, the ROSAT sequence identification number, and the SASS version used for the calibration of the data. The PSPC-derived X-ray positions are typically within $15^{\prime \prime}$ of the optical ones, so all the identifications are secure. Note that the exposure times are uncertain by about $4 \%$ due to a number of possible systematic errors, as described by Fiore et al. (1994). The angular radius from the target quasar which we used for 
TABLE 1

The Complete PG Quasar Soft X-RAy SURvey SAMPLE

\begin{tabular}{|c|c|c|c|c|c|c|c|}
\hline Quasar & Other Name & $z^{\mathbf{b}}$ & $m_{B}^{b}$ & $M_{B}^{\mathrm{b}}$ & $R^{\mathrm{c}}$ & $\begin{array}{c}N_{\mathrm{HI}}^{\mathrm{Gal}} \\
\left(10^{20} \mathrm{~cm}^{-2}\right)\end{array}$ & Reference for $N_{\mathrm{HI}}^{\mathrm{Gal}}$ \\
\hline PG 0947+396 & K $347-45$ & 0.206 & 16.40 & -24.06 & 0.25 & 1.37 & 1 \\
\hline PG $0953+414$ & $\mathrm{~K} 348-7$ & 0.239 & 15.05 & -25.73 & 0.44 & 1.27 & 1 \\
\hline PG $1001+054 \ldots \ldots \ldots \ldots$ & & 0.161 & 16.13 & -23.79 & 0.50 & 1.88 & 2 \\
\hline PG $1048+342 \ldots \ldots \ldots \ldots$ & & 0.167 & 15.81 & -24.19 & $\lesssim 0.10$ & 1.82 & 1 \\
\hline PG $1114+445^{\mathrm{d}}$ & & 0.144 & 16.05 & -23.63 & 0.13 & 1.82 & 1 \\
\hline PG $1115+407^{d}$ & & 0.154 & 16.02 & -23.81 & 0.17 & 1.82 & 1 \\
\hline PG $1116+215 \ldots \ldots \ldots \ldots$ & TON 1388 & 0.177 & 15.17 & -24.96 & 0.72 & 1.44 & 2 \\
\hline PG $1202+281 \ldots \ldots \ldots \ldots$ & GQ COM & 0.165 & 15.02 & -24.96 & 0.19 & 1.72 & 2 \\
\hline PG $1216+069^{d}$ & & 0.334 & 15.68 & -25.82 & 1.65 & 1.60 & 1 \\
\hline PG $1226+023^{d}$ & $3 \mathrm{C} 273$ & 0.158 & 12.86 & -27.02 & 1138 & 1.68 & 3 \\
\hline PG $1309+355^{\mathrm{d}}$ & TON 1565 & 0.184 & 15.45 & -24.76 & 18.0 & 1.00 & 1 \\
\hline PG $1322+659^{\mathrm{d}}$ & & 0.168 & 15.86 & -24.15 & 0.12 & 1.82 & 2 \\
\hline PG $1352+183^{d}$ & PB 4142 & 0.158 & 15.71 & -24.17 & 0.11 & 1.84 & 2 \\
\hline PG $1402+261 \ldots \ldots \ldots \ldots$ & TON 182 & 0.164 & 15.57 & -24.39 & 0.23 & 1.42 & 1 \\
\hline PG $1411+442$. & PB 1732 & 0.089 & 14.99 & -23.65 & 0.13 & 1.18 & 1 \\
\hline PG $1415+451^{\mathrm{d}}$ & & 0.114 & 15.74 & -23.43 & 0.17 & 1.10 & 1 \\
\hline PG $1425+267 \ldots \ldots \ldots \ldots$ & TON 202 & 0.366 & 15.67 & -26.03 & 53.6 & 1.54 & 2 \\
\hline PG $1427+480 \ldots \ldots \ldots \ldots$ & & 0.221 & 16.33 & -24.28 & 0.16 & 0.91 & 1 \\
\hline PG $1440+356 \ldots \ldots \ldots \ldots$ & Mrk 478 & 0.077 & 15.00 & -23.32 & 0.37 & 1.18 & 1 \\
\hline PG $1444+407 \ldots \ldots \ldots \ldots$ & & 0.267 & 15.95 & -25.07 & $\lesssim 0.08$ & 1.27 & 1 \\
\hline PG $1512+370^{d}$ & 4C 37.43 & 0.371 & 15.97 & -25.76 & 190 & 1.40 & 3 \\
\hline PG $1543+489^{d}$ & & 0.400 & 16.05 & -25.84 & 0.15 & 1.60 & 1 \\
\hline PG $1626+554$. & & 0.133 & 16.17 & -23.34 & 0.11 & 1.82 & 2 \\
\hline
\end{tabular}

a All PG sample quasars (i.e., $M_{B}<-23$ ) with $z \leq 0.400$ and $N_{\mathrm{HI}}^{\mathrm{Gal}}<1.9 \times 10^{20}$.

${ }^{b}$ From Schmidt \& Green 1983; $M_{B}$ calculated assuming $H_{0}=50 \mathrm{~km} \mathrm{~s}^{-1}$ and $q_{0}=0.5$.

c The ratio of radio $(6 \mathrm{~cm})$ to optical $(4400 \AA)$ flux, taken from Kellerman et al. 1989. Radio-loud quasars have $R \gtrsim 10$.

d Included in this paper.

REFERENCES.-(1) Stark et al. 1992. (2) Elvis et al. 1989. (3) Savage et al. 1993.

the extraction of source counts is $2^{\prime}-3^{\prime}$, which is the smallest radius at which the source counts saturate. The background counts were estimated using the source-free regions in an annulus around the central region with a width of typically $2^{\prime}-3^{\prime}$ centered on the target quasar. The typical number of counts per object is well over 1000, which allows an accurate determination of the spectral slope, as described below.

Model fits to the extracted number of source counts per pulse-invariant (PI) channel, $N_{\mathrm{ch}}^{\mathrm{ob}}$, were carried out using the XSPEC software package. We also used software developed by one of us (F. F.) to make fits using models not available in XSPEC, as further detailed below. Since all the observations were made after 1991 October 14, the 1993 January PSPC calibration matrix was used, and PI channels $1-10(E<0.11$
$\mathrm{keV})$ were ignored since they are not well calibrated. The bestfit model parameters are obtained by $\chi^{2}$ minimization. This method is valid when $N_{\mathrm{ch}}^{\mathrm{ob}}$ has a Gaussian probability distribution centered on $N_{\mathrm{ch}}^{\mathrm{mod}}$, the expected number of counts per channel. The actual probability distribution is, however, Poisson, and the Gaussian approximation becomes very inaccurate when $N_{\mathrm{ch}}^{\mathrm{mod}} \lesssim 10$. We therefore did not use the 256 PI channels, but rather used the standard division to 34 channels of merged PI channels, which provide a roughly constant $\Delta E / E$, where $\Delta E$ is the channel width. When necessary, we further merged nearby channels so that $N_{\mathrm{ch}}^{\mathrm{ob}} \geq 10$. A $1 \%$ error was added in quadrature to the statistical error in $N_{\mathrm{ch}}^{\mathrm{ob}}$, to take into account possible systematic calibration errors (G. Hasinger \& S. Snowden 1992, private communication).

TABLE 2

JOURNAL OF ROSAT PSPC OBSERVATIONS

\begin{tabular}{|c|c|c|c|c|c|c|c|c|c|}
\hline Quasar & $z$ & $\begin{array}{l}\text { Exposure }^{a} \\
\text { (s) }\end{array}$ & $\begin{array}{c}\text { Dates } \\
\text { (Start-End) }\end{array}$ & Counts ${ }^{b}$ & $\begin{array}{l}\text { Counts }^{b} \\
\left(s^{-1}\right)\end{array}$ & $r^{\mathrm{c}}$ & Offset $^{d}$ & Sequence Number & SASS Version \\
\hline PG $1114+445 .$. & 0.144 & 7373 & 1993 Jun $6.909-7.078$ & $777+30$ & 0.105 & $2^{\prime} 0$ & $15^{\prime \prime} 0$ & 700800 & 6.4 \\
\hline PG $1115+407 \ldots \ldots \ldots$ & 0.154 & 6495 & 1993 May $20.447-20.767$ & $1934 \pm 156$ & 0.298 & 2.5 & 14.8 & 700801 & 6.4 \\
\hline PG $1226+023 \ldots \ldots$ & 0.158 & 6243 & 1991 Dec $14.372-15.108$ & $38015 \pm 197$ & 6.089 & 3.0 & 8.0 & 700191 & 5.4 \\
\hline PG $1309+355 \ldots \ldots$ & 0.184 & 2528 & 1992 Jul $6.776-6.863$ & $593+25$ & 0.235 & 2.0 & 16.0 & 700802 & 5.7 \\
\hline PG $1322+659 \ldots \ldots$ & 0.168 & 8389 & 1992 Nov 30.038-Dec 1.932 & $4187+67$ & 0.499 & 2.5 & 5.4 & 700803 & 6.2 \\
\hline PG $1352+183 \ldots \ldots$. & 0.158 & 5521 & 1992 Jul $22.474-22.639$ & $2285 \pm 51$ & 0.414 & 3.0 & 13.1 & 700804 & 5.8 \\
\hline
\end{tabular}

a Uncertain by about $4 \%$ due to systematic errors.

b Net source counts in PI channels 11-245.

c Effective aperture for integration of counts.

d Offset of X-ray position from optical position. 


\subsection{A Single Power Law}

We fit each spectrum with a single power law of the form $f_{E}=e^{-N_{\mathrm{H}} \sigma_{E}} f_{0} E^{\alpha_{x}}$, where $f_{E}$ is the flux density, $\sigma_{E}$ is the absorption cross section per $\mathrm{H}$ atom (Morrison \& McCammon 1983), $f_{0}$ is the flux density and $1 \mathrm{keV}$, and $E$ is in units of $\mathrm{keV}$. We make three different fits for each object with (1) $N_{H}$ a free parameter, (2) $N_{\mathrm{H}}=N_{\mathrm{HI}}^{\mathrm{Gal}}$, and (3) $N_{\mathrm{H}}=N_{\mathrm{HI}}^{\mathrm{Gal}}$, and with $0.47 \leq E \leq 2.5 \mathrm{keV}$, i.e., using only the hard ROSAT band (channels 12-34).

A comparison of fits 1 and 2 allows us to determine whether there is evidence for a significant intrinsic absorption excess or emission excess relative to a single power-law fit with $N_{\mathrm{H}}=N_{\mathrm{HI}}^{\mathrm{Gal}}$. This comparison also allows us, as further shown in $\S 5.3 .3$, to determine whether the $21 \mathrm{~cm}$ measurement of $N_{\mathrm{HI}}^{\mathrm{Gal}}$ is a reliable measure of the Galactic soft X-ray opacity. A comparison of fits 2 and 3 allows us to look for a dependence of the power-law slope on energy. Fit 3 has the additional advantage of being less sensitive to a possible systematic error in $N_{\mathrm{HI}}^{\mathrm{Gal}}$ since the low-energy part of the spectrum, where the Galactic opacity is largest, is excluded (according to Morrison \& McCammon 1983, $\tau_{0.5 \mathrm{keV}}=N_{\mathrm{H}} / 1.36 \times 10^{21}$, thus $\tau_{0.5 \mathrm{keV}}<0.14$ in our sample). However, the smaller energy range available in fit 3 , together with the smaller number of counts, increases the statistical error in this case.

The results of fits 1-3 described above are given in Table 3.
For each fit we give the best-fitting spectral slope $\alpha_{x}$, the normalization of the power-law flux at $1 \mathrm{keV}\left(f_{0}\right)$, the best-fitting $N_{\mathrm{H}}$, the $\chi^{2}$ of the fit $\left(\chi_{\mathrm{fit}}^{2}\right)$, the number of degrees of freedom (dof), and the probability for $\chi^{2} \geq \chi_{\mathrm{fit}}^{2}$. The errors $\Delta \alpha_{x}$ and $\Delta N_{\mathrm{H}}$ in fit 1 were calculated by making a grid search for models with $\Delta \chi^{2}=2.30$, as appropriate for the $1 \sigma$ confidence level for two interesting parameters (e.g., Press et al. 1989).

In fits 2 and 3 we assume an $N_{\mathrm{HI}}^{\mathrm{Gal}}$ uncertainty of $\Delta N_{\mathrm{HI}}^{\mathrm{Gal}}=$ $2 \times 10^{19} \mathrm{~cm}^{-2}$, when using the $N_{\mathrm{HI}}^{\mathrm{Gal}}$ values from Stark et al. (1992). This uncertainty was estimated from a comparison of accurate $N_{\mathrm{H} I}^{\mathrm{Gal}}$ from Elvis et al. (1989) with the measurements of Stark et al. for $N_{\mathrm{HI}}^{\mathrm{Gal}} \leq 2 \times 10^{20} \mathrm{~cm}^{-2}$. We assume $\Delta N_{\mathrm{H}}=0$ when the small-beam $N_{\mathrm{HI}}^{\mathrm{Gal}}$ measurements by Elvis et al. and Savage et al. are used. Note that Elvis et al. estimate $\Delta N_{\mathrm{HI}}^{\mathrm{Gal}}=1$ $\times 10^{19} \mathrm{~cm}^{-2}$ for their measurements; however this is a general estimate for high Galactic latitudes, and the particularly low $N_{\mathrm{HI}}^{\mathrm{Gal}}$ selected here are likely to have a smaller uncertainty. The error on the slope $\Delta \alpha_{x}$ in fits 2 and 3 is calculated by requiring $\Delta \chi^{2}=1.0$ (i.e., $68 \%$ for one interesting parameter). Two components of this error, statistical and systematic, are indicated when an accurate $N_{\mathrm{HI}}^{\mathrm{Gal}}$ is not available. The first component is the statistical error (resulting from the finite number of counts), which is calculated as in fit 1 . The second, systematic component in $\Delta \alpha_{x}$ is calculated by repeating the power-law fits with $N_{\mathbf{H I}}^{\mathrm{Gal}}$ fixed at its upper and lower $1 \sigma$ limits. The systematic error in $\alpha_{x}$ is typically $0.08-0.1$, while the sta-

TABLE 3

Single POWER-LAW MOdels $\left(f_{E}=e^{-N_{H} \sigma_{E}} f_{0} E^{\alpha_{x}}\right)$

\begin{tabular}{|c|c|c|c|c|c|c|}
\hline Name & $\mathrm{Fit}^{\mathrm{a}}$ & $\alpha_{x}^{b}$ & $\begin{array}{c}f_{0}^{\mathrm{c}} \\
\left(10^{-4}\right)\end{array}$ & $\begin{array}{c}N_{\mathrm{H}}^{\mathrm{d}} \\
\left(10^{20} \mathrm{~cm}^{-2}\right)\end{array}$ & $\chi_{\mathrm{fii}}^{2} / \mathrm{dof}$ & $\operatorname{Pr}\left(\chi^{2} \geq \chi_{\mathrm{fit}}^{2}\right)$ \\
\hline \multirow[t]{3}{*}{ PG $1114+445 \ldots \ldots \ldots$} & 1 & $-1.13_{-0.31}^{+0.24}$ & $1.97 \pm 0.20$ & $1.04 \pm 0.62$ & $59.72 / 25$ & $1.1 \times 10^{-4}$ \\
\hline & 2 & $-1.50 \pm 0.06 \pm 0.09$ & $1.92 \pm 0.13 \pm 0.03$ & $1.82 \pm 0.2$ & $63.11 / 26$ & $6.3 \times 10^{-5}$ \\
\hline & 3 & $-0.17 \pm 0.24 \pm 0.01$ & $1.97 \pm 0.13 \pm 0.02$ & $1.82 \pm 0.2$ & $35.81 / 17$ & $4.8 \times 10^{-3}$ \\
\hline \multirow[t]{3}{*}{ PG $1115+407 \ldots \ldots$. } & 1 & $-1.77 \pm 0.17$ & $3.82 \pm 0.28$ & $1.48 \pm 0.36$ & $24.54 / 21$ & 0.27 \\
\hline & 2 & $-1.92 \pm 0.04 \pm 0.09$ & $3.81 \pm 0.19 \pm 0.02$ & $1.82 \pm 0.2$ & $32.73 / 22$ & 0.066 \\
\hline & 3 & $-1.86 \pm 0.14 \pm 0.05$ & $3.86 \pm 0.20 \pm 0.05$ & $1.82 \pm 0.2$ & $11.29 / 13$ & 0.59 \\
\hline \multirow[t]{3}{*}{ PG $1216+069 \ldots \ldots$} & 1 & $-1.519 \pm 0.20$ & $8.01 \pm 0.57$ & $1.96_{-0.43}^{+0.48}$ & $24.14 / 25$ & 0.51 \\
\hline & 2 & $-1.369 \pm 0.040 \pm 0.082$ & $7.93 \pm 0.38 \pm 0.09$ & $1.60 \pm 0.2$ & $25.74 / 26$ & 0.48 \\
\hline & 3 & $-1.435 \pm 0.13 \pm 0.036$ & $7.97 \pm 0.41 \pm 0.05$ & $1.60 \pm 0.2$ & $17.05 / 17$ & 0.45 \\
\hline \multirow[t]{3}{*}{ PG $1226+023 \ldots \ldots}$. & 1 & $-0.917 \pm 0.032$ & $177.6 \pm 2.0$ & $1.61 \pm 0.08$ & $74.10 / 29$ & $8.2 \times 10^{-6}$ \\
\hline & 2 & $-0.942 \pm 0.0077$ & $178.0 \pm 1.3$ & 1.68 & $76.09 / 30$ & $7.1 \times 10^{-6}$ \\
\hline & 3 & $-0.831 \pm 0.023$ & $177.0 \pm 1.3$ & 1.68 & $20.44 / 21$ & 0.49 \\
\hline \multirow[t]{3}{*}{ PG $1309+335 \ldots \ldots$} & 1 & $-1.725 \pm 0.31$ & $3.00 \pm 0.40$ & $1.43_{-0.50}^{+0.62}$ & $20.99 / 22$ & 0.52 \\
\hline & 2 & $-1.507 \pm 0.068 \pm 0.10$ & $3.02 \pm 0.26 \pm 0.02$ & $1.0 \pm 0.2$ & $22.63 / 23$ & 0.48 \\
\hline & 3 & $-1.422 \pm 0.25 \pm 0.044$ & $3.03 \pm 0.40 \pm 0.02$ & $1.0 \pm 0.2$ & $10.92 / 14$ & 0.69 \\
\hline \multirow[t]{3}{*}{ PG $1322+659 \ldots \ldots$} & 1 & $-1.967 \pm 0.13$ & $8.23 \pm 0.37$ & $2.49 \pm 0.31$ & $25.23 / 26$ & 0.51 \\
\hline & 2 & $-1.690 \pm 0.024$ & $8.19 \pm 0.23$ & 1.82 & $38.21 / 27$ & 0.057 \\
\hline & 3 & $-1.862 \pm 0.083$ & $8.10 \pm 0.24$ & 1.82 & $16.70 / 18$ & 0.54 \\
\hline \multirow[t]{3}{*}{ PG $1352+183 \ldots \ldots}$. & 1 & $-1.347 \pm 0.15$ & $7.83 \pm 0.44$ & $1.43_{-0.31}^{+0.34}$ & $20.27 / 24$ & 0.68 \\
\hline & 2 & $-1.524 \pm 0.034$ & $7.85 \pm 0.17$ & $1.84^{-0.31}$ & $23.61 / 25$ & 0.54 \\
\hline & 3 & $-1.266 \pm 0.11$ & $7.93 \pm 0.29$ & 1.84 & $10.84 / 16$ & 0.82 \\
\hline \multirow[t]{3}{*}{ PG $1415+451 \ldots \ldots}$. & 1 & $-1.591 \pm 0.13$ & $3.32 \pm 0.25$ & $0.67_{-0.19}^{+0.22}$ & $19.98 / 24$ & 0.70 \\
\hline & 2 & $-1.825 \pm 0.040 \pm 0.10$ & $3.26 \pm 0.18 \pm 0.06$ & $1.1 \pm 0.2$ & $27.97 / 25$ & 0.31 \\
\hline & 3 & $-1.589 \pm 0.14 \pm 0.049$ & $3.40 \pm 0.17 \pm 0.03$ & $1.1 \pm 0.2$ & $15.11 / 16$ & 0.52 \\
\hline \multirow[t]{3}{*}{ PG $1512+370 \ldots \ldots$} & 1 & $-1.409 \pm 0.17$ & $6.05 \pm 0.39$ & $1.87 \pm 0.39$ & $53.30 / 25$ & $8.2 \times 10^{-4}$ \\
\hline & 2 & $-1.210 \pm 0.037$ & $6.00 \pm 0.25$ & 1.4 & $57.40 / 26$ & $3.7 \times 10^{-4}$ \\
\hline & 3 & $-1.226 \pm 0.13$ & $6.05 \pm 0.26$ & 1.4 & $13.02 / 17$ & 0.73 \\
\hline \multirow[t]{3}{*}{ PG $1543+489 \ldots \ldots$} & 1 & $-2.573_{-0.31}^{+0.27}$ & $0.98 \pm 0.13$ & $2.67_{-0.56}^{+0.67}$ & $29.93 / 23$ & 0.15 \\
\hline & 2 & $-2.067 \pm 0.057 \pm 0.10$ & $1.02 \pm 0.08 \pm 0.02$ & $1.6 \pm 0.2$ & $39.77 / 24$ & 0.023 \\
\hline & 3 & $-2.174 \pm 0.20 \pm 0.03$ & $1.00 \pm 0.09 \pm 0.01$ & $1.6 \pm 0.2$ & $20.93 / 15$ & 0.14 \\
\hline
\end{tabular}

a The best-fit single-power-law model for (1) PI channels 11-245 and $N_{\mathrm{H}}$ a free parameter, (2) PI channels $11-245$ and $N_{\mathrm{H}}=N_{\mathrm{HI}}^{\mathrm{Gal}}$, (3) PI channels 47-245 and $N_{\mathrm{H}}=N_{\mathrm{HI}}^{\mathrm{Gal}}$.

b The first error is $1 \sigma$ for either two interesting parameters (fit 1) or one interesting parameter (fits 2 and 3 ). The second error (for fits 2 and 3 ) is the possible systematic error due to the uncertainty in $N_{\mathrm{HI}}^{\mathrm{Gal}}$.

c In units of $\mathrm{keV} \mathrm{cm}^{-2} \mathrm{~s}^{-1} \mathrm{keV}^{-1}$, or equivalently $6.626 \times 10^{-27} \mathrm{ergs} \mathrm{cm}^{-2} \mathrm{~s}^{-1} \mathrm{~Hz}^{-1}$, at $1 \mathrm{keV}$.

d In fit 1 the $H_{\mathrm{H}}$ error is $1 \sigma$ for two interesting parameters. In fits 2 and 3 the $N_{\mathrm{H}}$ error is assumed to be zero when a small-beam accurate $N_{\mathbf{H I}}^{\mathrm{Gal}}$ measurement is available, or \pm 0.2 when only the large-beam Stark et al. $N_{\mathbf{H I}}^{\mathrm{Gal}}$ measurement is available. 
tistical uncertainty is typically only $0.04-0.06$. This demonstrates the critical importance of having very accurate measurements of $N_{\mathrm{HI}}^{\mathrm{Gal}}$, i.e. to within $\pm 1 \times 10^{19} \mathrm{~cm}^{-2}$ or better, which is required in order to reduce the systematic uncertainty below the typical statistical uncertainty. Otherwise, one cannot make full use of high-S/N ROSAT spectra. Accurate $N_{\mathrm{HI}}^{\mathrm{Gal}}$ measurements, as were made by Elvis et al. (1989), will be obtained in the near future for all the objects in our sample and also for many other AGNs observed by ROSAT. These measurements will be reported in a future paper.

The observed and best-fit spectra for $N_{\mathrm{H}}=N_{\mathrm{HI}}^{\mathrm{Gal}}$ are displayed in Figure 1. There are three panels for each object. The top panel displays the observed count rate per $\mathrm{keV}$ as a function of channel energy; the histogram represents the expected rate from the best-fit power-law model with $N_{\mathrm{H}}=N_{\mathrm{HI}}^{\mathrm{Gal}}$ (fit 2). The middle panel displays $\Delta / \sigma$, where $\Delta=N_{\mathrm{ch}}^{\mathrm{ob}}-N_{\mathrm{ch}}^{\mathrm{mod}}$ and $\sigma$ is the standard error in $N_{\mathrm{ch}}^{\mathrm{ob}}$. This plot helps indicate what features in the spectrum are significant. The bottom panel displays the fractional deviations from the expected flux, or equivalently $\Delta / N_{\mathrm{ch}}^{\mathrm{mod}}$, which indicates the amplitude of the observed features.

As shown in Table 3, in seven of the 10 objects there are no significant deviations from a simple power-law fit (i.e., $\operatorname{Pr}\left(\chi^{2} \geq\right.$ $\left.\left.\chi_{\text {fit }}^{2}\right)>0.01\right)$. Figure 1 indicates that in these seven objects intrinsic features at $E<0.5 \mathrm{keV}$ must have an amplitude of less than about $20 \%$ of the continuum level (at the PSPC spectral resolution), and less than $30 \%-50 \%$ at $E>0.5 \mathrm{keV}$, where the

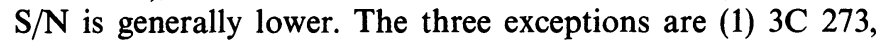
where significant deviations from a simple power law are present, but only at a level of about $5 \%-10 \%$; such small deviations are significant because of the very high $\mathrm{S} / \mathrm{N}$ spectrum of this object; (2) PG $1114+415$, where a strong absorption feature is suggested below $1 \mathrm{keV}$; and (3) PG $1512+370$, where there are significant deviations below $0.47 \mathrm{keV}$. Note that, although $\operatorname{Pr}\left(\chi^{2} \geq \chi_{\mathrm{fit}}^{2}\right)>0.01$ in the remaining seven objects (i.e., they do not deviate significantly from a singlepower-law fit), three of them have $\operatorname{Pr}\left(\chi^{2} \geq \chi_{\mathrm{fit}}^{2}\right)<0.1$, while less than one object $(0.7)$ is expected on the average. This excess of objects with a marginally high $\chi^{2}$ in our sample may suggest the presence of systematic features at a level comparable to the statistical error, or alternatively, it could result from small systematic calibration errors in the PSPC response function.

As mentioned above, a comparison of the free $N_{H}$ fit (fit 1) with the Galactic $N_{H}$ fit (fit 2) allows us to look for evidence for an absorption or an emission excess. We measure the statistical significance of the reduction in $\chi_{\mathrm{fit}}^{2}$ with the addition of $N_{\mathrm{H}}$ as a free parameter using the $F$-test (Bevington 1969). We find $\operatorname{Pr}=0.0026(F=12.98$ for 26 dof $)$ for $P G 1322+659$, and $\operatorname{Pr}=0.0092(F=9.84$ for 23 dof $)$ for PG $1543+489$, where $\operatorname{Pr}$ is the probability that the reduction in $\chi^{2}$ is not statistically significant (calculated using the FTEST routine in Press et al. 1989). An excess absorption, rather than emission, is suggested in both cases, with a value of $6.7 \times 10^{19} \mathrm{~cm}^{-2}$ in $P G$ $1322+659$, and $1.07 \times 10^{20} \mathrm{~cm}^{-2}$ in PG $1543+489$. In the other eight objects the free $N_{\mathrm{H}}$ fit does not provide a significant improvement (i.e., $\operatorname{Pr}>0.01$ ).

In all objects we find that a single power law provides a better fit to the region at $E \geq 0.47 \mathrm{keV}$ (fit 3), rather than to the complete spectrum (fit 2). In particular, we find that, in both $3 \mathrm{C}$ 273 and PG $1512+370$, a single power law describes well the hard ROSAT band, although it is clearly rejected as an acceptable fit for the full ROSAT band. The hard ROSAT band fit has a flatter slope that the overall spectral slope in PG
$1114+445,3 \mathrm{C} 273$, PG $1352+183$, and PG $1415+451$, and a steeper slope in PG $1322+659$. However, the average hard ROSAT band slope, $-1.52 \pm 0.40$ (excluding the unacceptable fit to PG $1114+445)$, is practically identical to the average full ROS AT band slope, $-1.56 \pm 0.36$.

\subsection{Energy-dependent Power-Law Slope}

As mentioned above, a comparison of fits 1 and 2, or fits 2 and 3 , allows us to look for curvature in the power-law spectra. However, a more direct way to look to curvature is by fitting a power-law model which includes an additional curvature term. We fitted all objects with the following power-law model

$$
f_{E}=e^{-N_{\mathrm{H}} \sigma_{E}} f_{0} E^{\alpha(E)},
$$

where

$$
\alpha(E)=\alpha_{0}+\beta \log E ;
$$

i.e., we fit a power law with a slope which varies linearly with $\log E$ (or a parabola, rather than a straight line, in the $\left(\log f_{E}\right.$, $\log E$ )-plane). Note that $\beta$ equals the change in $\alpha$ over one decade in $E$. A similar parameterization was used by Schwartz \& Tucker (1988) in the context of AGNs and the X-ray background.

Other commonly used spectral forms that go beyond a single power-law form are a broken law, two power laws, a power law + blackbody, or a power law + an optically thin free-free component. All these models share the disadvantage of introducing two, rather than one, additional free parameters. This inevitably results in a significantly larger uncertainty in the best-fit value for each of the fit parameters.

Energy-dependent power-law fits are not available in the standard X-ray spectral analysis packages (e.g., PROS, XSPEC). These fits were therefore made using the general model-fitting software developed by one of us (F. F.), which includes this option.

The results of the energy-dependent power-law fit are given in Table 4. For each object we give the best-fit parameters, the fit $\chi^{2}$, the number of dof, the $F$ statistics $\left(F_{\mathrm{fit}}\right)$, and the statistical significance of $F_{\mathrm{fit}}\left(\operatorname{Pr}\left(F \geq F_{\mathrm{fit}}\right)\right)$, where $F_{\mathrm{fit}}$ is the reduction of $\chi_{\text {fit }}^{2}$ in Table 4 compared with fit 2 in Table 3 (a single power law with $\left.N_{\mathrm{H}}=N_{\mathrm{HI}}^{\mathrm{Gal}}\right)$ and $\operatorname{Pr}\left(F \geq F_{\mathrm{fit}}\right)$ is the probability that the $\alpha(E)$ model does not provide a significantly better fit. The errors in the best-fit parameters are $1 \sigma$ for two interesting parameters $\left(\alpha_{0}\right.$ and $\left.\beta\right)$. All fits assume $N_{\mathrm{H}}=N_{\mathrm{HI}}^{\mathrm{Gal}}$.

In PG $1114+445$ and 3C 273 the curved power-law model provides a significantly better fit, but in both cases the new $\chi^{2}$ is still high and more complicated models are suggested. None of the remaining eight objects has a significant improvement of $\chi^{2}$ at better than the $1 \%$ level. The best-fit value for the curvature term $\beta$ is within $2 \sigma$ of zero in these eight objects. Note, however, that four of the eight objects have $\operatorname{Pr}\left(F \geq F_{\text {fit }}\right)<$ $10 \%$, while less than one object is expected on the average. This might indicate the presence of a small, but real, intrinsic curvature in some of the objects.

We did not fit a model with $\alpha(E)$ and a free $N_{\mathrm{H}}$, since the low spectral resolution of the PSPC, and the available $\mathrm{S} / \mathrm{N}$, do not allow a high- $N_{\mathrm{H}}$, large- $\beta$ (i.e., very concave) model to be discriminated from a low $-N_{\mathrm{H}}$ and small- $\beta$ model.

\subsection{The Objects with Unacceptable Fits}

As mentioned above, fits 1 and 2 were unacceptable in PG $1114+445$, 3C 273, and PG $1512+370$. An acceptable model is required for carrying out the correlation analysis described below, and the model selected for each object is described here. 

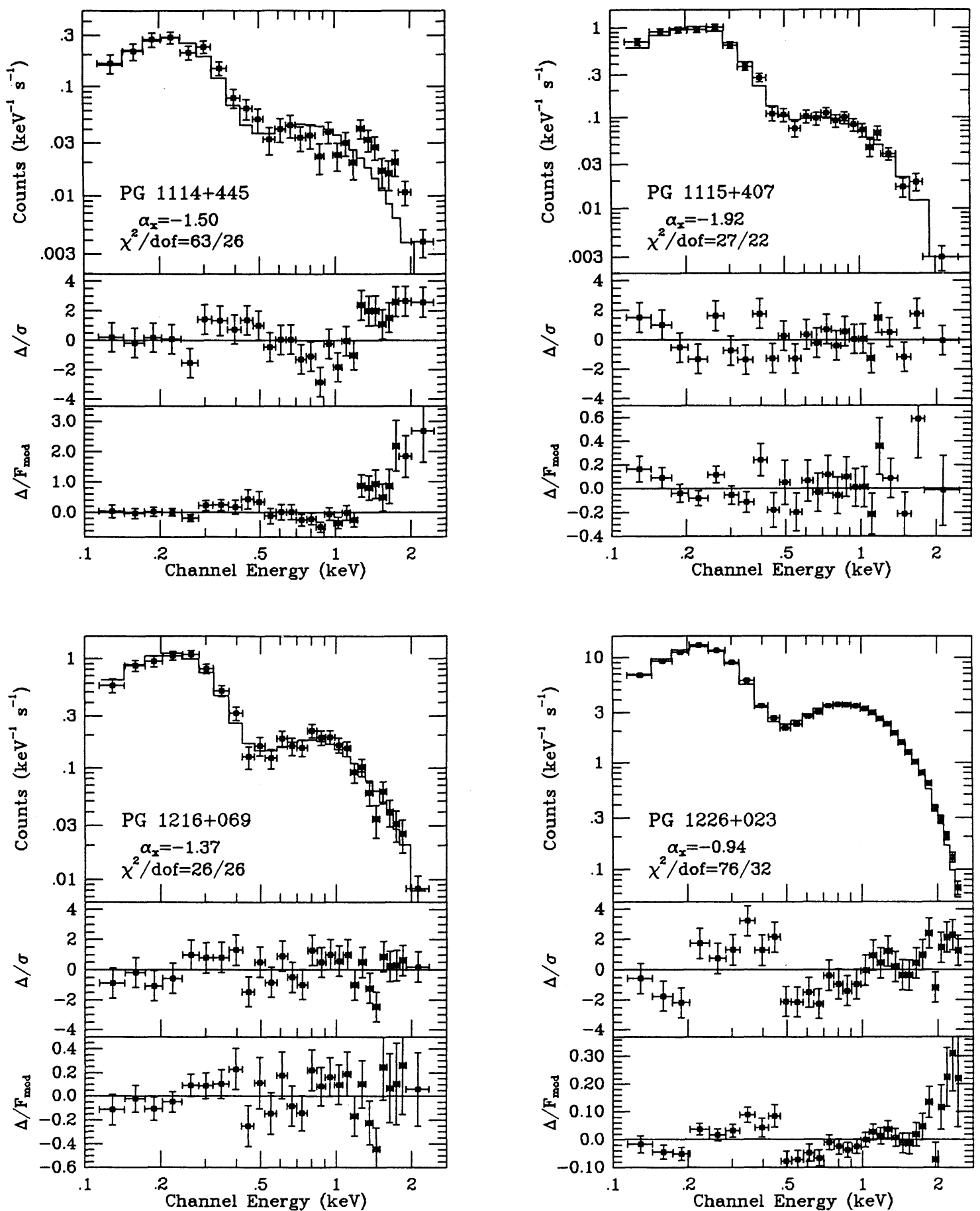

FIG. 1.-Observed vs. single power-law fit PSPC spectra of the 10 quasars. The top panel for each object shows the observed count rate (points with error bars) and the expected count rate using the best-fit single power-law model with $N_{\mathrm{HI}}^{\mathrm{Gal}}$ (solid-line histogram), the middle panel shows the observed minus expected flux in units of standard deviations, and the bottom panel for each object shows the fractional deviations from the expected flux. The best-fit $\alpha_{x}$, the fit $\chi^{2}$, and the number of dof are indicated for each object. Seven of the 10 spectra are consistent with a simple power law. Intrinsic features must have an amplitude or less than $\sim 30 \%$ of the expected continuum level. The three exceptions are: 3 C 273, where the very high S/N indicates significant deviations from a simple power law, but only at a level of about $5 \%-10 \%$; PG $1114+415$, were a strong absorption edge is suggested (see text); and PG $1512+370$, where there are significant deviations below $0.5 \mathrm{keV}$.

In the case of PG $1114+445$ we find that a single power law + an absorption edge provides an acceptable fit. We use the simple power law + edge model described in Fiore et al. (1993). The best-fit parameters (as in Table 3) are

$$
\begin{gathered}
\alpha_{x}=-0.88_{-0.38}^{+0.29}, \quad f_{0}=4.77 \pm 1.2, \\
E_{\text {edge }}=0.76 \pm 0.07 \mathrm{keV}, \quad N_{\mathbf{H}}^{1}=(7.1 \pm 0.3) \times 10^{21},
\end{gathered}
$$

where we assume $N_{\mathrm{H}}=N_{\mathrm{HI}}^{\mathrm{Gal}}, N_{\mathrm{H}}^{1}$ is the additional column of cold gas which absorbs above $E_{\text {edge }}$ (rest-frame energy), and all the errors are $1 \sigma$. This model gives $\chi^{2} /$ dof $=20.65 / 23$. These parameters imply an edge with an optical depth of about 3.1 at $0.76 \mathrm{keV}$ (Morrison \& McCammon 1983). This edge is reminiscent of the O-edges reported in PSPC spectra of 3C 351 (Fiore et al. 1993), MCG -6-30-15 (Nandra \& Pounds 1992), 

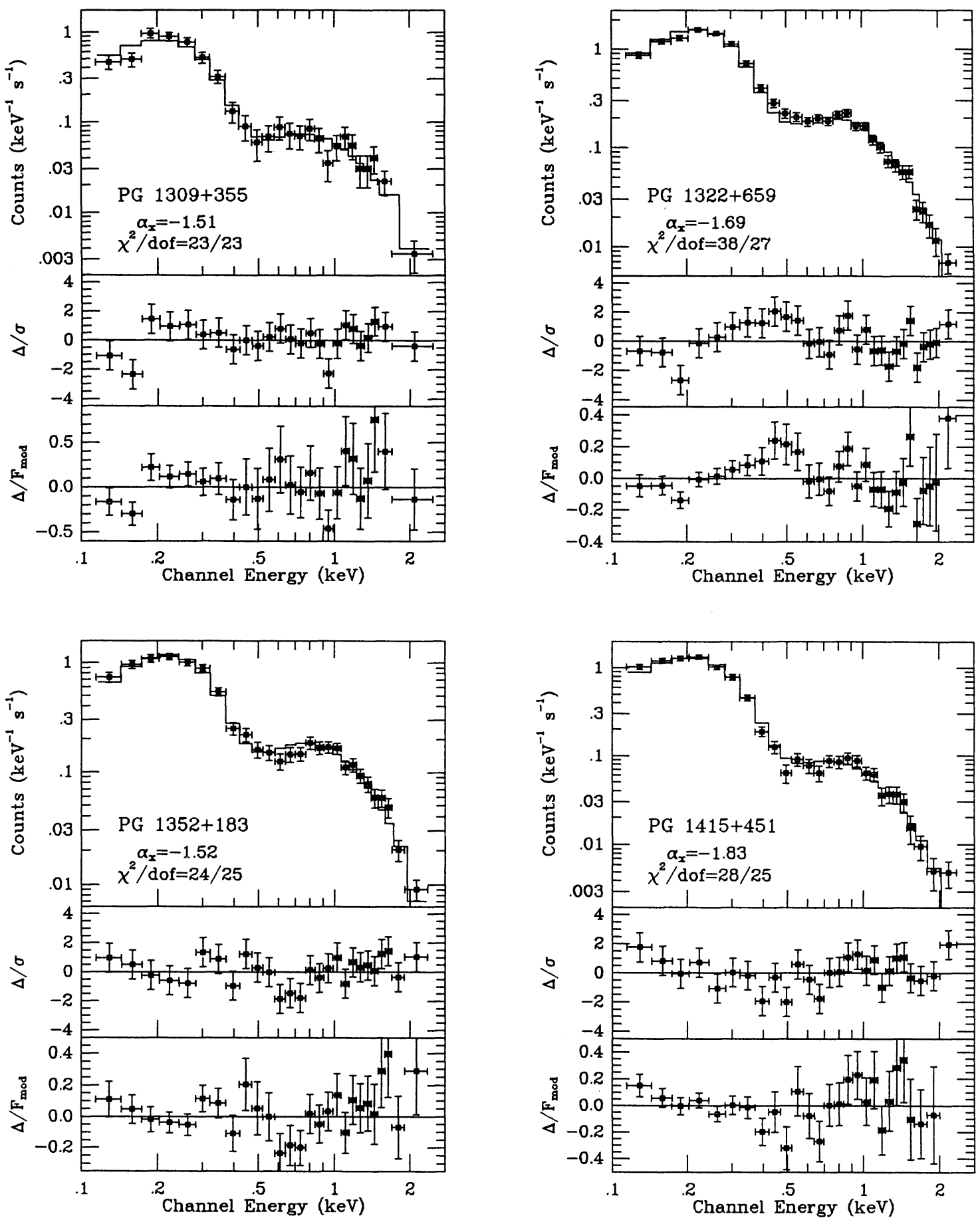

FIG. 1-Continued

and NGC 3783 (Turner et al. 1993b). This edge model is rather simplified, and a more detailed and more physical analysis of the absorption properties of PG $1114+445$ will be given in a forthcoming paper.

In the case of $3 \mathrm{C} 273$, the high $\chi^{2}$ is not due to largeamplitude deviations from a power-law model, but rather to deviations at a level of only $5 \%-10 \%$, which are significant because of the very high $\mathrm{S} / \mathrm{N}$ available $(38,015$ counts). Had $3 \mathrm{C}$ 273 been observed with only 2000 counts, as is typical for the rest of the sample, these intrinsic features would have been undetectable, and the single power law would have been acceptable. We therefore use the parameters of fit 2 for $3 \mathrm{C} 273$ (in Table 3) for the correlation analysis described below. It is not clear to us at this stage whether the features in the spectrum of 3C 273 are indeed real, or whether they are induced by systematic calibration errors (see Fiore et al. 1994).

The fit for PG $1512+370$ could not be improved by a simple and plausible modification of the single power-law fit, as expected for the complicated residuals structure (Fig. 1). However, the fit above $0.47 \mathrm{keV}$ in PG $1512+370$ is acceptable 

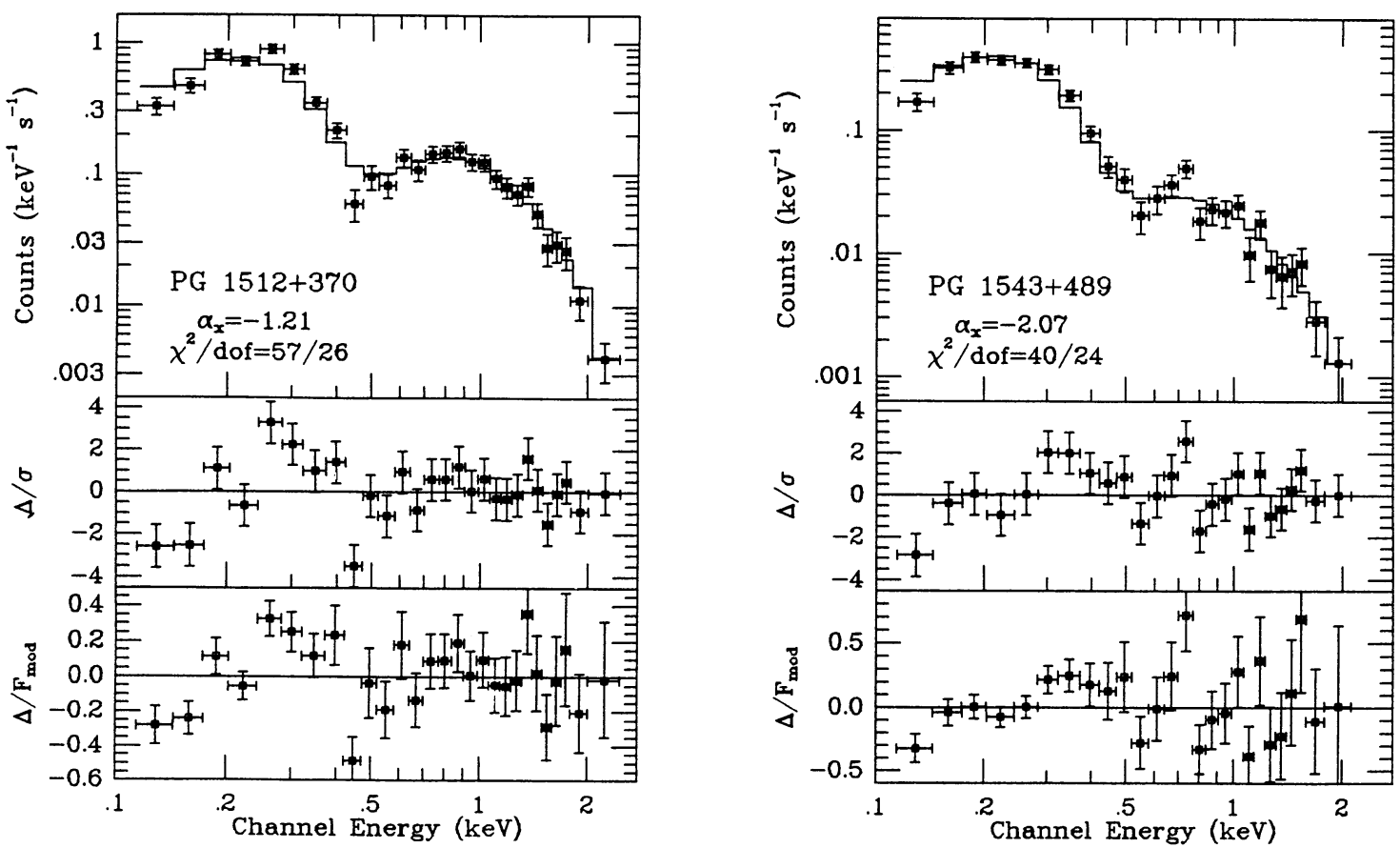

FIG. 1-Continued

$\left(\chi^{2} /\right.$ dof $\left.=0.76\right)$, which indicates the structure is confined to the soft ROSAT band. In this object we use the parameters of fit 3 $(E>0.47 \mathrm{keV})$ for the correlation analysis. The apparent features at $E<0.47 \mathrm{keV}$ might be related to the presence of an associated metal line absorption system in this object (see Bahcall et al. 1993), as in the case of the quasar 3C 351 described by Fiore et al. (1993) and Mathur et al. (1993).

\section{CORRELATION ANALYSIS}

As mentioned above, the PG quasar sample has been extensively explored, and a large database with the continuum and emission line properties of the PG quasars is already available. We used this database to study correlations of the continuum and emission line properties with the soft X-ray fluxes and slopes measured here. Table 5 presents all the rest-frame continuum and emission line parameters used for the correlation analysis. The near-IR and optical fluxes were taken from Neugebauer et al. (1987). The X-ray continuum parameters are either from fit 2 , or as indicated in $\S 3.3$. The spectral slopes are defined as $\alpha_{o}=\log \left(f_{15} / f_{14.5}\right) / 0.5, \alpha_{o x}=\log \left(f_{17.685} / f_{15}\right) / 2.685$, $\alpha_{o s}=\log \left(f_{16.861} / f_{15}\right) / 1.861$ (optical to soft X-ray slope), and $\alpha_{\text {irs }}=\log \left(f_{16.861} / f_{14.25}\right) / 2.611$ (IR to X-ray slope). The flux subscript indicates $\log \mathrm{Hz}\left(10^{14.25} \mathrm{~Hz}=1.69 \mu \mathrm{m}, 10^{14.5}\right.$ $\mathrm{Hz}=0.95 \mu \mathrm{m}, 10^{15} \mathrm{~Hz}=3000 \AA, 10^{16.861} \mathrm{~Hz}=0.3 \mathrm{keV}$, and $\left.10^{17.865} \mathrm{~Hz}=2 \mathrm{keV}\right)$. We have chosen to measure the flux at $1.69 \mu \mathrm{m}$ and $0.3 \mathrm{keV}$, since these are the longest IR wavelength and lowest X-ray rest-frame energy for which the flux can be determined for all objects by interpolation. Similarly, $3000 \AA$ and $2 \mathrm{keV}$, are the shortest wavelength and highest energy available (the PSPC response formally extends to $2.5 \mathrm{keV}$; however the sensitivity above $2 \mathrm{keV}$ is very low).

The emission-line parameters are taken from the extensive, high-quality, spectroscopic database of the PG quasars obtained by Boroson \& Green (1992). The emission-line luminosities were calculated using the equivalent widths (EWs) from Boroson \& Green, and the continuum fluxes from Neugebauer et al. Boroson \& Green specify the measurement error only for the Fe II EW. For the other lines we assume an EW error of $0.5 \AA$, which is half the smallest EW reported. This

TABLE 4

ENERGY-DEPENDENT POWER-LAW MODELS $\left(f_{E}=e^{-N_{H} \sigma_{E}} f_{0} E^{\alpha_{0}+\beta \log E}\right)$

\begin{tabular}{|c|c|c|c|c|c|c|c|}
\hline Name & $\alpha_{0}$ & $\beta$ & $\begin{array}{c}f_{0}^{\mathbf{a}} \\
\left(10^{-4}\right) \\
\end{array}$ & $\chi_{\mathrm{fit}}^{2} / \mathrm{dof}$ & $\operatorname{Pr}\left(\chi^{2} \geq \chi_{\mathrm{fit}}^{2}\right)$ & $F_{\mathrm{fit}}^{\mathrm{b}}$ & $\operatorname{Pr}\left(F \geq F_{\mathrm{fit}}\right)$ \\
\hline PG $1114+445 \ldots \ldots$ & $-0.54_{-0.23}^{+0.26}$ & $1.85 \pm 0.52$ & $2.27 \pm 0.30$ & $40.00 / 25$ & 0.029 & 23.11 & $1.2 \times 10^{-4}$ \\
\hline PG $1115+407$. & $-1.82 \pm 0.20$ & $0.20 \pm 0.37$ & $5.66 \pm 0.43$ & $25.80 / 21$ & 0.21 & 6.93 & 0.031 \\
\hline PG $1216+069$. & $-1.44 \pm 0.15$ & $-0.20 \pm 0.36$ & $16.3 \pm 1.5$ & $24.86 / 25$ & 0.47 & 0.88 & 0.71 \\
\hline PG $1226+023 \ldots \ldots$ & $-0.88 \pm 0.03$ & $0.14 \pm 0.06$ & $230.8 \pm 2.9$ & $62.25 / 29$ & $3.2 \times 10^{-4}$ & 13.84 & $1.7 \times 10^{-3}$ \\
\hline PG $1309+335 \ldots \ldots$ & $-1.56_{-0.31}^{+0.37}$ & $-0.10 \pm 0.59$ & $4.67 \pm 0.62$ & $22.51 / 22$ & 0.43 & 0.12 & 1.00 \\
\hline PG $1322+659 \ldots \ldots \ldots$ & $-1.89 \pm 0.13$ & $-0.42 \pm 0.24$ & $13.0 \pm 0.58$ & $30.37 / 26$ & 0.25 & 7.84 & 0.019 \\
\hline PG $1352+183 \ldots \ldots$ & $-1.33 \pm 0.14$ & $0.43 \pm 0.28$ & $10.8 \pm 0.7$ & $17.96 / 24$ & 0.80 & 5.65 & 0.052 \\
\hline PG $1415+451 \ldots \ldots$ & $-1.55 \pm 0.19$ & $0.49 \pm 0.32$ & $4.27 \pm 0.34$ & $22.53 / 24$ & 0.55 & 5.44 & 0.057 \\
\hline PG $1512+370 \ldots \ldots$ & $-1.27 \pm 0.13$ & $-0.19 \pm 0.31$ & $12.5 \pm 1.0$ & $56.16 / 25$ & $3.5 \times 10^{-4}$ & 1.24 & 0.55 \\
\hline PG $1543+489 \ldots \ldots$. & $-2.37_{-0.25}^{+0.30}$ & $-0.77_{-0.70}^{+0.60}$ & $3.17 \pm 0.36$ & $36.20 / 23$ & 0.039 & 3.57 & 0.14 \\
\hline
\end{tabular}

${ }^{a}$ In units of $\mathrm{keV} \mathrm{cm}^{-2} \mathrm{~s}^{-1} \mathrm{keV}^{-1}$ at $1 \mathrm{keV}$.

${ }^{\mathrm{b}}$ Compared with the fixed power-law model (fit 2 in Table 3). 

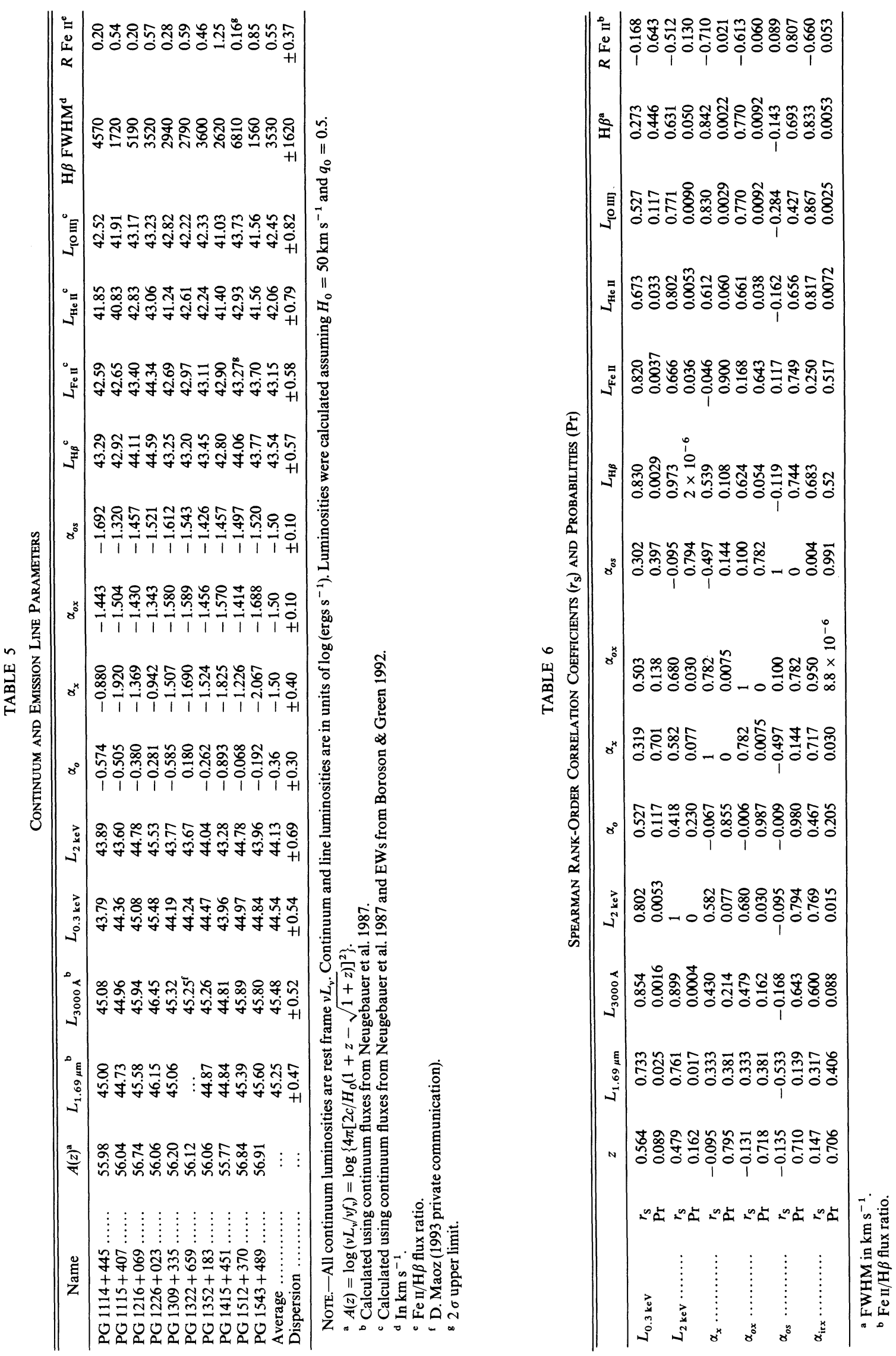
error is negligible for $\mathrm{H} \beta$, but can be very large for [O III] in a few cases. All luminosities were calculated assuming $H_{0}=50$ $\mathrm{km} \mathrm{s}^{-1}$ and $q_{0}=0.5$, and in Table 5 we also specify

$$
\mathrm{A}(z)=\log \left(v L_{v} / v f_{v}\right)=\log \left\{4 \pi\left[2 c / H_{0}(1+z-\sqrt{1+z})\right]^{2}\right\},
$$

where $L_{v}$ is the luminosity density, which allows the observed flux density $\left(f_{v}\right)$ to be immediately inferred.

We searched for correlations between the X-ray luminosities, the various spectral slopes, and the emission line parameters which Boroson \& Green described as significantly correlated. We also searched for some of the correlations reported in earlier studies (see discussion in $\$ 5.3 .1$ ). The correlations' significance was tested using the Spearman rank-order correlation coefficient $\left(r_{s}\right)$. This statistical correlation test is sensitive to any monotonic relation between the two variables and is thus more general than the Pearson correlation coefficient $(r)$ which tests for the presence of linear correlations only. The Spearman $r_{\mathrm{S}}$ has the additional advantage that it is insensitive to outliers, which generally have a significant effect on the value of $r$. A summary of all correlation coefficients and their significance is given in Table 6.

The small size of the sample analyzed here implies that only relatively strong correlations can be reliably detected. Specifically, a correlation will appear significant in our sample (i.e., $\operatorname{Pr} \leq 0.01$ ) only for $r_{\mathrm{s}} \geq 0.76$. Below we mainly concentrate on the strongest correlations found. These correlations are likely to remain significant when the complete sample of 23 quasars is analyzed. A more comprehensive correlation analysis will be presented in a forthcoming paper where the complete sample will be analyzed.

\subsection{The Near-IR to $X$-Ray Energy Distribution}

Figure 2 presents the IR to X-ray energy distribution of the 10 quasars. The $0.3-3 \mu \mathrm{m}$ continua were taken from Neugebauer et al. (1987). The $0.2-2 \mathrm{keV}$ continua are the best-fit single-power-law model to the PSPC spectra with $N_{\mathrm{H}}=N_{\mathrm{HI}}^{\mathrm{Gal}}$, or as indicated in $\S 3.3$. The $\pm 1 \sigma$ slopes are plotted using 0.6 $\mathrm{keV}$ as the pivot point. The error bar at $0.6 \mathrm{keV}$ represents the $1 \sigma$ error in the flux. The flux error includes the error in the observed number of counts, and the $4 \%$ error in the net exposure time $(\S 3)$ which was added in quadrature. UV spectra are being obtained for our complete sample, and they will be presented in a future paper.

Note the overall similarity of the X-ray to optical ratios, despite the considerable range in local slopes in both the optical and in the X-ray, and the fact that the optical and X-ray observations were taken about $10 \mathrm{yr}$ apart. The well-known near-IR spectral break at $\log v \sim 14.4-14.5$ (e.g., Sanders et al. 1989 ) is clearly apparent in all spectra.

A comparison of the spectral energy distributions of the quasars is given in Figure 3. The upper panel shows the absolute luminosities, and the lower panel the luminosity normalized to unity at $\log v=14.25$ (PG $1322+659$ was excluded from the lower panel since near-IR photometry is not available for it). The dispersion in the log of normalized $0.3 \mathrm{keV}$ flux is 0.28 , versus a dispersion of 0.38 at $2 \mathrm{keV}$. The dispersions are 0.21 versus 0.41 when the highly uncertain $\alpha_{x}$ of PG $1114+445$ is excluded. The small dispersion in the normalized $0.3 \mathrm{keV}$ flux implies that $f_{1.69 \mu \mathrm{m}}$ and $f_{0.3 \mathrm{kev}}$ are correlated and proportional. Using the luminosities given in Table 5 we find an average spectral slope from $1.69 \mu \mathrm{m}$ to $0.3 \mathrm{keV}\left\langle\alpha_{\mathrm{irs}}\right\rangle=-1.26$ \pm 0.11 (or $\left\langle\alpha_{\text {irs }}\right\rangle=-1.23 \pm 0.08$, when PG $1114+445$ is excluded). The small dispersion in $\left\langle\alpha_{\text {irs }}\right\rangle$ implies that $f_{0.3 \mathrm{keV}}$ can be typically predicted to better than a factor of 2 once $f_{1.69 \mu \mathrm{m}}$ is known.

$L_{1.69 \mu \mathrm{m}}$ appears to be correlated with both $L_{0.3 \mathrm{keV}}$ and $L_{2 \mathrm{keV}}\left(r_{\mathrm{S}}=0.733, \operatorname{Pr}=0.025\right.$, and $\left.\left.r_{\mathrm{S}}=0.761, \operatorname{Pr}=0.017\right)\right)$. However, $L_{2 \mathrm{kev}}$ increases more rapidly than $L_{1.69 \mu \mathrm{m}}$, which results in the larger scatter in the normalized $f_{2 \mathrm{kev}}$.

The lower panel of Figure 3 suggests that the X-ray flux at 2 $\mathrm{keV}$ weakens relative to the optical, as the fitted soft X-ray slope steepens. This is confirmed by the presence of a significant correlation between $\alpha_{x}$ and $\alpha_{o x}\left(r_{S}=0.782, \operatorname{Pr}=0.0075\right)$, and the absence of a significant correlation between $\alpha_{x}$ and $\alpha_{o s}$ $\left(r_{\mathrm{s}}=-0.497, \operatorname{Pr}=0.15\right)$.

We note in passing that the local spectral slopes, $\alpha_{x}$ and $\alpha_{o}$, appear to be completely uncorrelated $\left(r_{\mathrm{S}}=-0.067, \operatorname{Pr}=0.85\right)$.

\subsection{Correlations with Emission Line Properties}

\subsubsection{Line Luminosity versus $X$-Ray Luminosity}

Figure 4 presents the correlations of the hard X-ray luminosity, defined here as $L_{2 \mathrm{kev}}$, or the soft X-ray luminosity, $L_{0.3 \mathrm{keV}}$, with the luminosities of $\mathrm{H} \beta$, [O III], He II, or Fe II. The value of $r_{\mathrm{S}}$ and the two-sided significance level $(\mathrm{Pr})$ of $r_{\mathrm{S}}$ are indicated above each panel. The correlation of $L_{\mathrm{H} \beta}$ and $L_{2 \mathrm{keV}}$ $\left(r_{\mathrm{S}}=0.973, \operatorname{Pr}=2 \times 10^{-5}\right)$ is the strongest correlation we find among all the various parameters given in Table 5. $L_{\mathbf{H} \beta}$ is also strongly correlated with $L_{15}\left(r_{\mathrm{S}}=0.939\right)$ and $L_{14.5}\left(r_{\mathrm{S}}=0.933\right)$, and it is well correlated with $L_{14.25}\left(r_{\mathrm{S}}=0.867\right)$ and $L_{0.3 \mathrm{keV}}$ $\left(r_{\mathrm{S}}=0.830\right)$.

$L_{[\mathrm{O} \text { III] }}$ is well correlated with $L_{2 \mathrm{keV}}\left(r_{\mathrm{S}}=0.771\right)$, but it is not significantly correlated with $L_{0.3 \mathrm{kev}}\left(r_{\mathrm{S}}=0.527\right)$. He II also shows a stronger correlation with the hard X-ray luminosity. Note that the error in $L_{\mathrm{He} \mathrm{II}}$ can be significantly larger than indicated in Figure 4, in particular when it is strongly blended with the Fe II emission (see Fig. 1 in Boroson \& Green). Fe II is the only one of these four lines which is better correlated with the soft X-ray luminosity $\left(r_{\mathrm{S}}=0.820\right)$.

\subsubsection{Line Properties versus Soft $X$-Ray Slope}

Figure 5 displays the line emission properties which most strongly correlate with $\alpha_{x}$. These properties are the FWHM of $\mathrm{H} \beta, L_{[\mathrm{OII}]}$, and the $\mathrm{Fe} \mathrm{II} / \mathrm{H} \beta$ flux ratio. The left-hand panels show these correlations using the $\alpha_{x}$ obtained in fit $2\left(N_{\mathrm{H}}=\right.$ $N_{\mathrm{HI}}^{\mathrm{Gal}}$, or as in $\left.\S 3.3\right)$, and the right-hand panels show the correlations using $\alpha_{x}$ obtained in fit 1 (free $N_{H}$ ). The strength and significance of each correlation are indicated in each panel. All the correlations with a fixed $N_{\mathbf{H}}$ are significantly stronger than the correlations with a free $N_{\mathrm{H}}$.

Two objects, PG $1114+445$ and $3 \mathrm{C} 273$, fall significantly away from the suggested fixed $N_{\mathrm{H}}$ correlation. In the case of PG $1114+445$, the error in $\alpha_{x}$ is very large (it has to be fit by a more complicated model, see $\S 3.3$ ), and its $\alpha_{x}$ deviates by only 1.2-1.8 $\sigma$ from the correlation defined by the other eight objects. The other object, with the very small error bar, is $3 \mathrm{C}$ 273. Repeated ROSAT observations have shown that this quasar has a variable $\alpha_{x}$ (see Staubert 1992; Staubert et al. 1992). The ROSAT all-sky survey (RASS) observation of $3 \mathrm{C}$ 273 gives $\alpha_{x}=-1.29$ (for $N_{\mathrm{H}}=N_{\mathrm{H} 1}^{\mathrm{Gal}}$; Staubert 1992). This value of $\alpha_{x}$ is in significantly closer agreement with the correlation defined by the other eight quasars. If the two deviant objects are ignored, then the correlations shown in Fig. 5 become significantly stronger. For $\mathrm{H} \beta$ FWHM versus $\alpha_{x}$ we find $r_{\mathrm{S}}=0.976\left(\mathrm{Pr}=3.3 \times 10^{-5}\right)$, for $\left.L_{[\mathrm{O}} \mathrm{III}\right]$ versus $\alpha_{x}$ we find $r_{\mathrm{S}}=0.929\left(\mathrm{Pr}=8.6 \times 10^{-4}\right)$, and for $\mathrm{Fe} \mathrm{II} / \mathrm{H} \beta$ versus $\alpha_{x}$ we find $r_{\mathrm{S}}=-0.881\left(\mathrm{Pr}=3.9 \times 10^{-3}\right)$. 

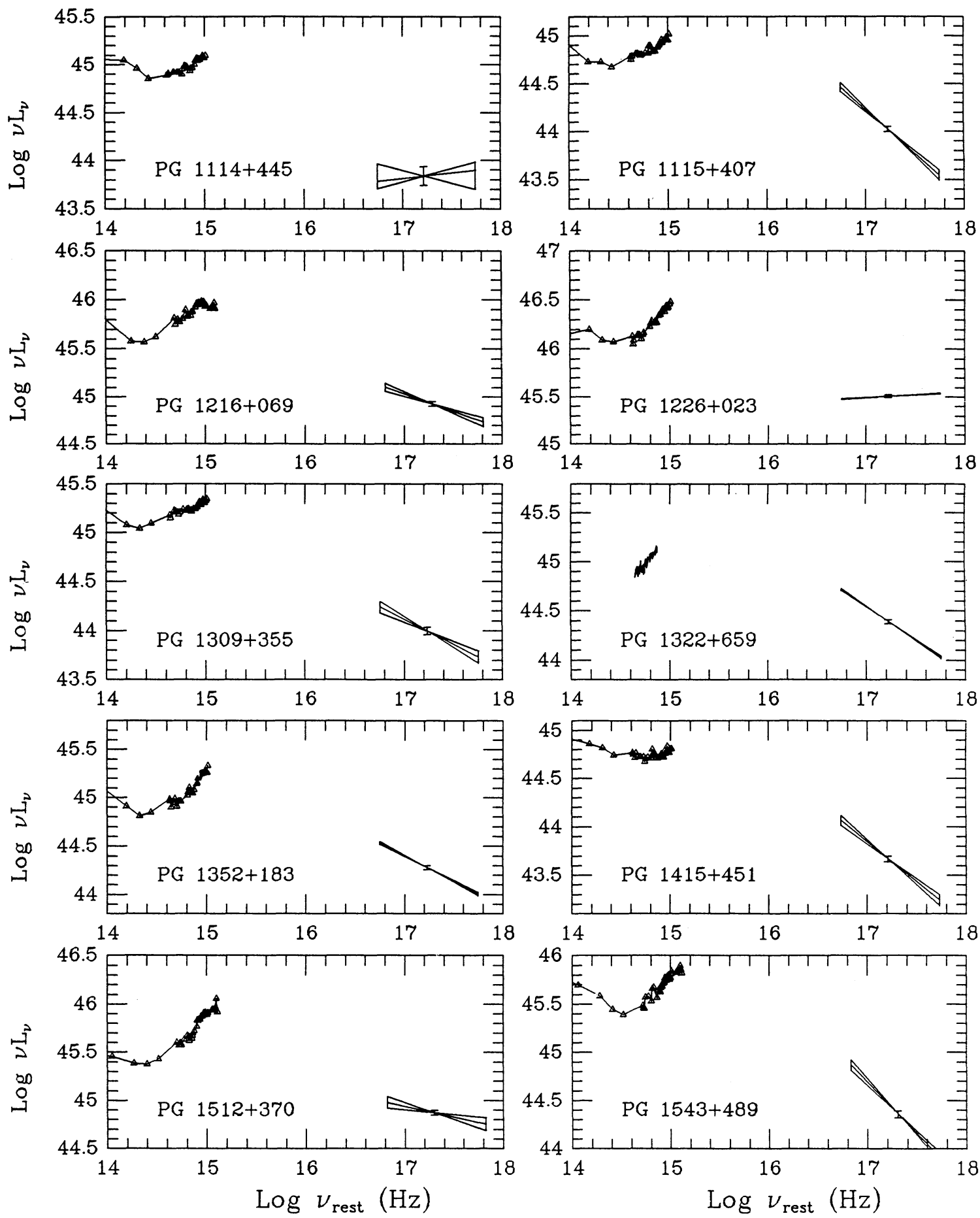

FIG. 2.-IR to X-ray energy distribution of the 10 quasars. The $0.3-3 \mu \mathrm{m}$ continua are from Neugebauer et al. (1987). The $0.2-2 \mathrm{keV}$ continua are the acceptable models to the PSPC spectra, assuming $N_{\mathrm{HI}}^{\mathrm{Gal}}$ (see $\$ 3.1$ and 3.3). The $\pm 1 \sigma$ slopes are plotted using $0.6 \mathrm{keV}$ at the pivot point. The error bar at $0.6 \mathrm{keV}$ represents the $1 \sigma$ error in the flux. Note the overall similarity of the ratio of X-ray to optical flux, despite the considerable range in local spectral slopes in the optical and in the $\mathrm{X}$-ray, and the large time span $(\sim 10 \mathrm{yr})$ between the optical and the $\mathrm{X}$-ray observations.

\subsection{Redshift-induced Correlations}

Our sample is selected from the flux-limited PG sample. The observed $3000 \AA$ flux of nine of the 10 quasars (excluding $3 C$ 273) ranges over a factor of 2 only, while their inferred optical luminosity ranges over a factor of 13 . This results in a significant optical luminosity versus redshift correlation. However, no flux limit was imposed at either the IR or the X-ray range; thus the continuum-continuum correlations found here are not expected to be induced by redshift correlations. To verify this point we calculated the partial correlation coefficients for a fixed redshift (using eq. [27.5] in Kendall \& Stuart 1977). In all cases where the correlations were significant (i.e., $r_{\mathrm{s}}>0.76$ ), the 

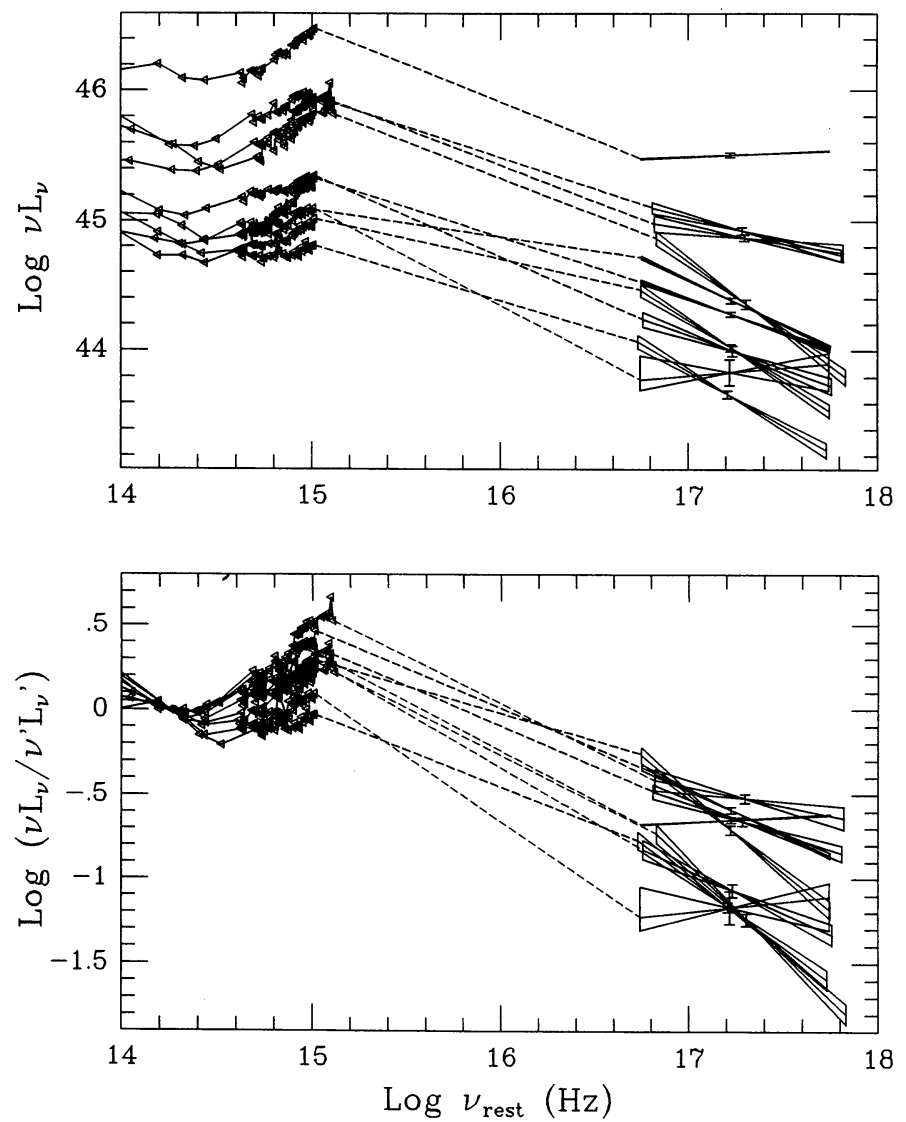

Fig. 3.-Comparison of the spectral energy distribution of the 10 quasars: upper panel, the absolute luminosity; lower panel, the luminosity (or flux) normalized to unity at $\log v=14.25$ (the longest wavelength available for all objects). Note that the spread in the normalized flux at $0.2 \mathrm{keV}$ is smaller than at $2 \mathrm{keV}$. Thus a steep $\alpha_{x}$ is generally associated with a weak hard X-ray component, rather than a strong soft X-ray excess. The small spread in the normalized soft X-ray flux, together with the general lack of a significant near-IR variability, indicate that the soft X-ray flux typically does not vary by more than $\pm 50 \%$. (The object with the largest error in $\alpha_{x}$ is PG $1114+445$, which is fit with a power law + absorption edge.)

partial correlations for a fixed $z, r_{\mathrm{S}, z}$, had a rather similar value. For example, for $\alpha_{x}$ versus $\alpha_{o x}$ we get $r_{\mathrm{S}, z}=0.783$, where $r_{\mathrm{S}}=$ 0.782 , for $\alpha_{x}$ versus H $\beta$ FWHM $r_{\mathrm{S}, z}=0.866$, where $r_{\mathrm{S}}=0.742$, and for $L_{\mathrm{H} \beta}$ versus $L_{2} \mathrm{keV} r_{\mathrm{S}, z}=0.968$, where $r_{\mathrm{S}}=0.973$.

\section{DISCUSSION}

\subsection{The Soft $X$-Ray Spectral Shape}

\subsubsection{The Spectral Slope}

The significantly improved energy resolution and sensitivity of the PSPC, compared with the earlier IPC observations on Einstein and the LE observations on EXOSAT, allows us to determine the best-fit $\alpha_{x}$ with a typical statistical error of only $2 \%-4 \%$, which is about an order of magnitude smaller than the typical error available for quasars with earlier instruments (e.g., Comastri et al. 1992; Shastri et al. 1993).

We find an average spectral index $\left\langle\alpha_{x}\right\rangle=-1.50 \pm 0.40$ for our 10 quasars, where the error is the dispersion about the mean $\left(\alpha_{x}\right.$ are from Table 5). Walter \& Fink (1993) analyzed the PSPC spectra of 58 AGNs observed in the RASS. Although Walter \& Fink describe their objects as Seyfert 1-type AGNs, 24 of their AGNs are in fact classified as quasars by Véron-
Cetty \& Veron (1991). Using the values tabulated for the 24 quasars by Walter \& Fink we find $\left\langle\alpha_{x}\right\rangle=-1.57 \pm 0.30$, for $N_{\mathrm{H}}=N_{\mathrm{H} 1}^{\mathrm{Gal}}$. The sample of Walter \& Fink is not well defined. However, since it is drawn from the brightest RASS AGNs, it is probably dominated by soft X-ray bright AGNs. The similarity of $\left\langle\alpha_{x}\right\rangle$ obtained here and by Walter \& Fink, suggests that optically selected quasars and soft X-ray bright quasars have a similar distribution of $\alpha_{x}$.

Three of our quasars can be defined as radio-loud (where we use $R \geq 10$; see Table 1). Radio-loud quasars are known to have a systematically flatter X-ray slope at higher X-ray energies (Wilkes \& Elvis 1987). For the seven radio-quiet quasars in our sample we obtain $\left\langle\alpha_{x}\right\rangle=-1.61 \pm 0.15$, and for the three radio-loud quasars we get $\left\langle\alpha_{x}\right\rangle=-1.23 \pm 0.20$, where the errors represent the uncertainty in the value of the mean, rather than the dispersion about the mean. It therefore appears that the trend observed at harder X-rays also extends to the $R O S A T$ band, although a larger number of objects is required in order to reliably established this effect.

The X-ray spectra of nine of the 10 quasars described here (excluding PG $1114+445$ ) are consistent with a single-powerlaw shape at $0.15-2 \mathrm{keV}$. Deviations from a single power law are typically $30 \%$ or less. Small, but statistically significant, deviations from a single power-law shape are present in the spectra of 3C 273 and of PG $1512+370$. In PG $1114+445$ the deviations are significantly larger than $30 \%$, and the spectrum is well described by a power law + an absorption edge. Walter $\&$ Fink also concluded that most of the AGNs in their sample are consistent with a featureless power-law continuum, although the average $S / N$ in their spectra is significantly smaller since their spectra were obtained in the RASS, which in most cases allows only relatively short exposure times. Thus, the possible limits on intrinsic features in the RASS sample quasars are weaker.

The $\left\langle\alpha_{x}\right\rangle$ found by the PSPC on ROSAT is significantly steeper than that indicated by earlier studies of quasars. Wilkes \& Elvis (1987) and Masnou et al. (1992) analyzed the soft X-ray properties of quasars using the Einstein IPC $0.2-3.5 \mathrm{keV}$ spectra and found excess emission above the best-fitting power law. They found the excess starts below $\sim 0.6 \mathrm{keV}$ and it typically dominates the power-law component below $\sim 0.3 \mathrm{keV}$. The IPC observations indicated an $\alpha_{x} \sim-1$ in radio-quiet quasars and $\sim-0.5$ in radio-loud quasars, which is consistent with the hard X-ray slope of quasars (i.e., above $2 \mathrm{keV}$ ) as found by EXOSAT (Lawson et al. 1992) and by Ginga (Williams et al. 1992). A similar soft excess below $1 \mathrm{keV}$ was also found in the combined LE + ME EXOSAT spectra of quasars by Comastri et al. (1992) and Saxton et al. (1993). A soft excess was also found in Einstein and EXOSAT spectra of Seyfert 1 galaxies (Turner \& Pounds 1989; Kruper, Urry, \& Canizares 1990). As mentioned in $\S 3.1$, the ROSAT spectra are mostly well fit by a single power law with a steeper slope, and no soft emission excess, or absorption excess, is apparent.

The ROSAT spectra of quasars clearly indicate that the soft $\mathrm{X}$-ray slope is steeper than the hard X-ray slope. However, they suggest a steepening by $\Delta \alpha \sim 0.5$ below $\sim 1 \mathrm{keV}$, rather than $\Delta \alpha \gtrsim 1$ below $\sim 0.5 \mathrm{keV}$ as suggested earlier (e.g., Turner \& Pounds 1989; Masnou et al. 1992; Comastri et al. 1992). We can clearly rule out the possibility that the spectral steepening results from a soft component confined to $E \leq 0.5 \mathrm{keV}$, since, as mentioned in $\S 3.1$, the average spectral slope in the hard ROSAT band is also $\sim-1.5$. The difference between the ROSAT results and the earlier Einstein and EXOSAT results is 

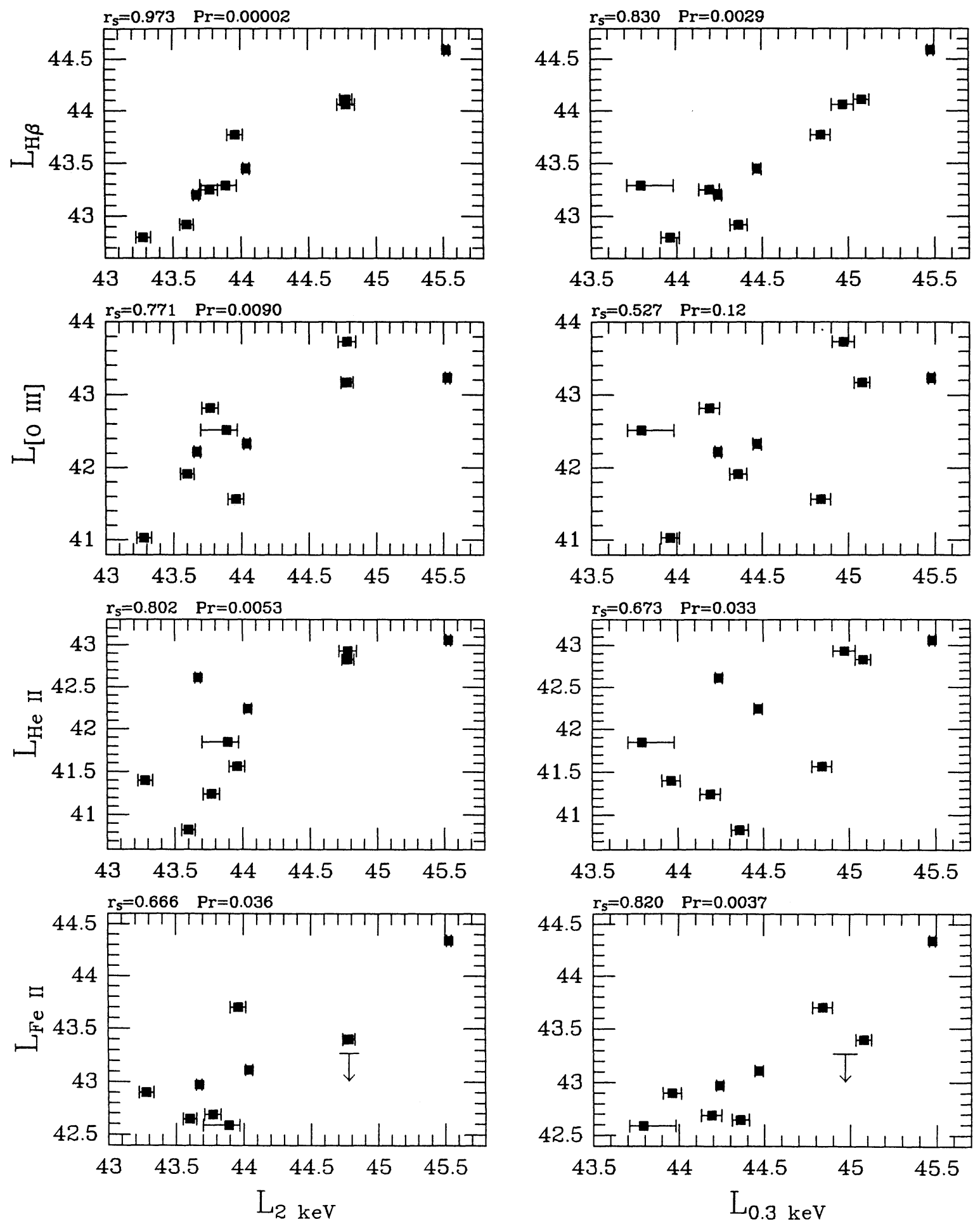

FIG. 4.-Correlations of the line luminosity vs. X-ray luminosity. The Spearman rank-order correlation coefficient $\left(r_{\mathbf{S}}\right)$ and the two-sided significance level (Pr) are

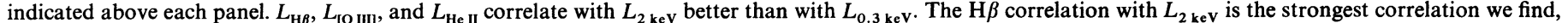

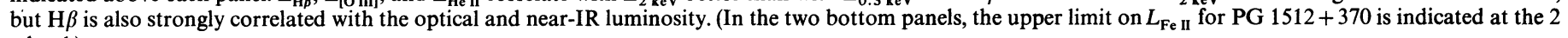
$\sigma$ level.) 

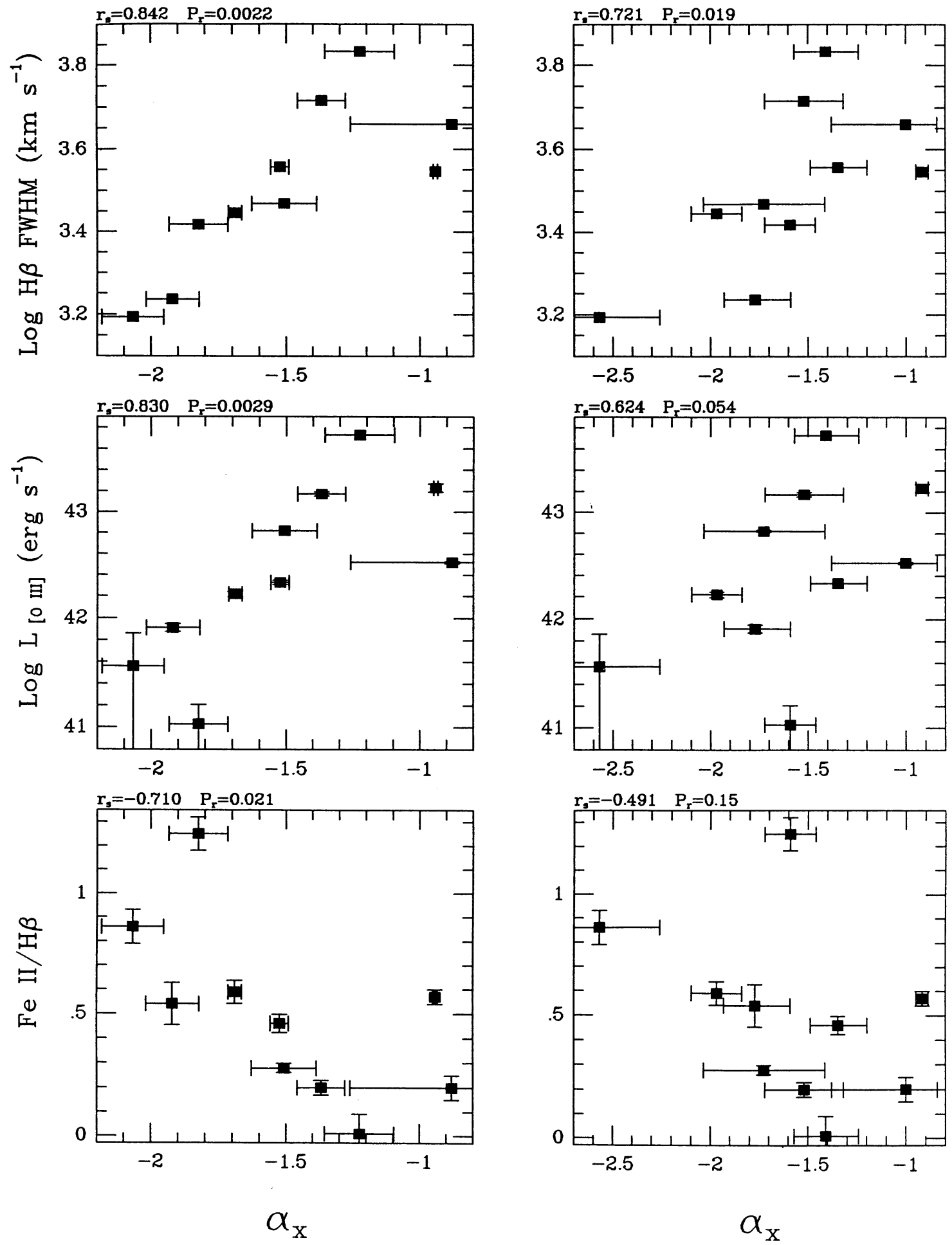

Fig. 5.-Emission properties which most strongly correlate with $\alpha_{x}\left(r_{\mathrm{s}}\right.$ and $\operatorname{Pr}$ are as in Fig. 4): left-hand panels, best-fit $\alpha_{x}$ with $N_{\mathrm{H}}$ fixed at the Galactic value; right-hand panels, best-fit $\alpha_{x}$ with $N_{\mathrm{H}}$ as a free parameter. All correlations with a fixed $N_{\mathrm{H}}$ are significantly stronger. This indicates that the neutral $\mathrm{H}$ column in the Galaxy is a good measure of the soft X-ray opacity. The presence of these correlations indicates that $\alpha_{x}$ does not vary significantly in most objects. The deviant object at $\alpha_{x}=-0.94$ with a very small error bar is 3 C 273, which has a variable $\alpha_{x}$ (the RASS measurement of 3 C 273 gives $\alpha_{x}=-1.29$ ). 
somewhat surprising in view of the large overlap in energy range covered by these instruments. The apparently different IPC and PSPC results might be traced back to the combined effect of the significant differences in the instruments' response functions (the PSPC being more sensitive below $\sim 0.5 \mathrm{keV}$ ), together with significant structure in the continuum shape, or it might reflect some calibration errors (see discussion in Fiore et al. 1994).

\subsubsection{Intrinsic Absorption}

As described in $\$ 3.1$, intrinsic absorption is suggested in the spectra of PG $1322+659$ and PG $1543+489$. There are, however three arguments which indicate that this intrinsic absorption might not be real: (1) Although the free $N_{\mathrm{H}}$ provides a lower $\chi^{2}$, the $\chi^{2}$ of the fit with $N_{\mathrm{H}}=N_{\mathrm{HI}}^{\mathrm{Gal}}$ is acceptable $(\operatorname{Pr}>0.01)$ in these two objects. (2) Quasars which are fit using the 1993 January PSPC response matrix (used here) tend to have an excess absorption of about $\Delta N_{\mathrm{H}} \sim 5 \times 10^{19} \mathrm{~cm}^{-2}$ over the Galactic value, while the fit using the 1992 March matrix indicates absorption which is generally in better agreement with the Galactic value, as shown by Fiore et al. (1994). A similar systematic bias toward higher than Galactic absorption is likely to be present in our sample as well. (3) As shown in $\S$ 4.2.2, and further discussed below, there are significant correlations between $\alpha_{x}$ and other emission line parameters, when $N_{\mathrm{H}}=N_{\mathrm{HI}}^{\mathrm{Gal}}$ is assumed. These correlations are much weaker when $N_{\mathrm{H}}$ is allowed to vary, which strongly suggests a general lack of intrinsic absorption in our sample (see further discussion in $\S 5.3 .3$ ). One should note, however, that these arguments are all indirect, and the possibility of intrinsic absorption in these quasars is not ruled out.

\subsubsection{The Spectral Curvature}

Since it is clear that $\alpha_{x}$ changes from hard to soft X-rays in AGNs, it is possible that $\alpha_{x}$ changes within the PSPC band itself. We searched for a curvature in the spectra by fitting an energy-dependent spectral slope of the form $\alpha(E)=\alpha_{0}+\beta \log E$ (see $\S 3.2$ ). As shown in Table $4, \beta$ deviates from zero by more than $2 \sigma$ only in PG $1114+445$ and in $3 C 273$. In the case of PG $1114+445, \beta=1.85 \pm 0.52$, which implies an extreme $(\sim 1)$ hard $X$-ray slope. The large deviations from a singlepower law model in PG $1114+445$ most likely result from an absorption edge, rather than a very large curvature, as mentioned in $\S 3.3$. In the case of $3 C 273$, the available $S / N$ is very high and we find $\beta=0.14 \pm 0.06$. Note, however, that $\chi_{\mathrm{fit}}^{2}$ is still too high.

The expected curvature can be estimated in 3C 273 by comparing the soft and the hard X-ray slopes, which gives the average curvature $\langle\beta\rangle$ over the 0.2 to $10 \mathrm{keV}$ range. Staubert (1992) describes simultaneous ROSAT and Ginga observations of $3 \mathrm{C} 273$ which indicate a steepening from -0.56 to -0.84 from the hard to the soft X-ray bands. The mean energy of these bands differs by about an order of magnitude, which therefore implies $\langle\beta\rangle \sim 0.28$. The fact that $\langle\beta\rangle$ is significantly larger than $\beta$ suggests the presence of a spectral "break" in the transition from the soft to the hard X-ray band. Note that inspection of the fractional deviations from the simple powerlaw fit to the very high-S/N spectrum of 3C 273 (Fig. 1), suggests a large excess, i.e., possibly a spectral break, at $E \gtrsim 1.5$ $\mathrm{keV}$ (observed frame).

We do not have a reliable estimate of the hard X-ray slope for other objects in our sample. We can calculate the expected $\langle\beta\rangle$ assuming our objects have the average hard $\mathrm{X}$-ray slope of quasars. The average hard $\mathrm{X}$-ray slope in radio-quiet quasars in $\left\langle\alpha_{x}\right\rangle \gtrsim-0.9$ to -1.0 (Comastri et al. 1992; Lawson et al. 1992; Williams et al. 1992), which implies $\langle\beta\rangle \gtrsim 0.5-0.6$ from $0.2-2 \mathrm{keV}$ to $2-10 \mathrm{keV}$. The measured $\beta$ is consistent with this value in PG $1115+407$, PG $1352+183$, and PG $1415+451$. However, when averaging over the whole sample (excluding PG $1114+445$ ) we find $\beta_{\mathrm{av}}=-0.04 \pm 0.13$ (error in the mean), which is significantly smaller than $\langle\beta\rangle$. This would suggest, assuming our quasars have the average hard X-ray slope, that the change in $\alpha_{x}$ in quasars is not continuous, but rather that there is a spectral break at $E \sim 1.5-2 \mathrm{keV}$.

As mentioned in $\S 5.1 .2$, there is a possible bias in the PSPC response matrix at low $E$ (effective area is overestimated). This bias tends to lower the value of $\beta$ when $N_{\mathrm{H}}=N_{\mathrm{HI}}^{\mathrm{Gal}}$ is imposed. To compensate for such a possible bias we fitted our spectra with the $\alpha(E)$ model, setting $N_{\mathrm{H}}=N_{\mathrm{HI}}^{\mathrm{Gal}}+5 \times 10^{9} \mathrm{~cm}^{-2}$. This revised fit yielded $\beta_{\mathrm{av}}=0.37 \pm 0.13$, which is consistent with $\langle\beta\rangle$, in which case there is a smooth change of slope from hard $(2-10 \mathrm{keV})$ to soft $(0.2-2 \mathrm{keV}) \mathrm{X}$-rays. We therefore conclude that given the possible calibration errors of the PSPC at low $E$, we cannot reliably descriminate $\beta=0$ from $\beta=\langle\beta\rangle$.

\subsection{Implications of the Continuum-Continuum Correlations}

Continuum-continuum luminosity correlations in quasars are generally strong. This reflects the fact that the overall energy distribution of quasars, excluding the radio emission, does not show a large dispersion and does not show a strong dependence on the absolute luminosity.

Here we find a rather small scatter in the near-IR to X-ray flux ratios. For example, $f_{0.3 \mathrm{kev}}$ in our sample can be predicted to within a factor of 2 , once $f_{1.69 \mu \mathrm{m}}$ is given. The presence of a significant correlation between the IR and X-ray fluxes, and luminosities, has been widely reported (e.g., Kriss, Canizares, \& Ricker 1980; Lawrence \& Elvis 1982; Carleton et al. 1987; Williams et al. 1992). The scatter in the correlations found here is significantly smaller. This probably reflects the homogeneity of our sample, compared with earlier samples, as all our quasars are optically selected and at $z \leq 0.400$. Elvis et al. (1994) have demonstrated that the host galaxy in lowluminosity quasars $\left(M_{V} \gtrsim-24\right)$ can dominate the measured H-band (observed $1.65 \mu \mathrm{m}$ ) flux. If the host galaxy contribution in the $\mathrm{H}$ band is not well correlated with the soft X-ray flux, as is likely to be the case, then its effect is to decrease the observed $f_{1.69 \mu \mathrm{m}}$ versus $f_{0.3 \mathrm{kev}}$ correlation. Thus, the correlation excluding the host galaxy contribution is likely to be somewhat stronger than found here.

Large-amplitude soft X-ray variability is well documented in quasars and Seyfert galaxies (e.g., Elvis et al. 1991, and references therein). The X-ray variability power spectrum, $P(f)$, of Seyfert galaxies at $f=10^{-5}-10^{-3} \mathrm{~Hz}$ can be described as $P(f) \sim f^{-1}$ to $f^{-2}$ (e.g., Lawrence et al. 1987; McHardy 1990; Lawrence \& Papadakis 1993). The small spread in the normalized soft X-ray flux found here, together with the general lack of significant near-IR variability in AGNs (Cutri et al. 1985; Neugebauer et al. 1989), implies that the soft X-ray flux does not vary by typically more than a factor of 2 from the mean over timescales shorter than a few years. This confirms the result of Zamorani et al. (1984) who found using the Einstein IPC that although nine out of the 12 quasars in their sample varied on a timescale of a year, the amplitude of variability was always less than a factor of 2 . These results imply that $P(f)$ in quasars does not continue to increase with increasing timescales but rather flattens out somewhere between $f \sim 10^{-5}$ and $f \sim 10^{-8}$, as found by McHardy (1990) in two Seyfert galaxies, NGC 5506 and NGC 4151. 
The average $3000 \AA$ to $2 \mathrm{keV}$ rest-frame spectral slope in our sample is $\left\langle\alpha_{o x}\right\rangle=-1.50 \pm 0.10$, which is identical to $\left\langle\alpha_{x}\right\rangle$ (§ 5.1.1). Brunner et al. (1992) also found $\left\langle\alpha_{o x}\right\rangle=\left\langle\alpha_{x}\right\rangle$ in ROSAT spectra of flat-radio-spectrum quasars, and a similar relation was suggested in Seyfert 1 galaxies by Turner, George, \& Mushotzky (1993a). However, this does not imply the presence of a single power-law component extending from the optical and the soft X-rays, since the local optical slope in quasars is generally much flatter. For example, Francis et al. (1991) found a median $\alpha_{o}=-0.32$ for 688 optically selected quasars, while in our sample $\left\langle\alpha_{o}\right\rangle=-0.36 \pm 0.30$ (Table 5). The values of $\left\langle\alpha_{o x}\right\rangle$ and $\left\langle\alpha_{x}\right\rangle$, together with $\left\langle\alpha_{o}\right\rangle$, imply a significant spectral steepening, or a "break," in the intrinsic spectral slope somewhere in the 1000-100 $\AA$ range.

As mentioned in $\S 4.1$, we find a significant correlation of $\alpha_{x}$ with $\alpha_{o x}$. This correlation can be interpreted in one of two ways. If the PSPC spectrum is actually a sum of a steep powerlaw component at low energy and a flatter component at higher energy, then a steeper observed $\alpha_{x}$ implies a weaker hard component. This would be consistent with earlier suggestions, based upon Einstein spectra of quasars (Wilkes \& Elvis 1987), that the strength of the flat, harder component is linked to the radio emission. Alternatively, the PSPC spectrum may include a single component whose slope varies with $\alpha_{o x}$, in which case the pivot point for the change in slope is within the PSPC energy range and this point is closer to 0.3 than to 2 $\mathrm{keV}$.

The $\alpha_{x}$ versus $\alpha_{o x}$ correlation is similar to the result of Walter $\&$ Fink who found a strong correlation between $\alpha_{x}$ and the $1375 \AA$ to $2 \mathrm{keV}$ flux ratio. We note, however, that the sample of Walker \& Fink is strongly biased toward AGNs with a large number of RASS counts (their principal selection criteria). This implies that objects with a steep $\alpha_{x}$ are detectable to lower integrated X-ray flux levels in the RASS, thus possibly inducing some of the correlation seen in their sample. Note that a small bias in the estimate of the $2 \mathrm{keV}$ flux is also possible in our sample due to the likely change in $\alpha_{x}$ at $E>2 \mathrm{keV}$. However, since the rest frame $2 \mathrm{keV}$ corresponds to observed 1.4-1.8 keV in our sample, where the PSPC still has a significant area, we do not expect this bias to be above $\sim 20 \%$ (the typical S/N at 1.4-1.8 keV; see Fig. 1).

The presence of an apparently significant $\alpha_{x}$ versus $\alpha_{o x}$ correlation, together with the apparent lack of a significant $\alpha_{x}$ versus $\alpha_{o s}$ correlation ( $\$ 4.1$ ), suggest that a steep $\alpha_{x}$ is mostly due to a weak hard X-ray component rather than a strong soft X-ray excess (see also Fig. 3). An opposite conclusion was drawn by Puchnarewicz et al. (1992), who studied the Einstein IPC spectra of the ultrasoft survey (USS) sample of AGNs, which is described by Córdova et al. (1992). The USS AGNs were selected to have a large fraction of their X-ray flux, as measured by the IPC, below $0.5 \mathrm{keV}$ (note that, due to an error in the Einstein IPC reprocessing, some of the USS quasars do not follow the original selection criteria; see further details in Thompson et al. 1994). Puchnarewicz et al. concluded that two-thirds of their objects had a normal ratio optical to hard $\mathrm{X}$-ray flux ratio and that the steep $\alpha_{x}$ in these objects therefore results from a strong soft component. The contrasting results found here might be due to a difference in the properties of optically versus soft X-ray selected quasars.

Both thin and thick accretion disk models predict the soft $\mathrm{X}$-ray flux to be strongly dependent on inclination, being brighter in edge-on thin disks, or face-on thick disks (e.g., Laor $\&$ Netzer 1989; Madau 1988). The optical flux is likely to have a weaker inclination dependence in thick disks, or have the reverse inclination dependence in thin disks. These models therefore suggest a large range of $\alpha_{o s}$ for accretion disks seen at a range of inclinations. Note also that thin accretion disks also predict large variations in the ratio of soft X-ray to optical flux among quasars due to differences in the accretion rate and the central black hole mass, independent of inclination effects (e.g., Laor \& Netzer 1989). These predictions contrast with the small range of optical to soft X-ray flux observed here $\left(\left\langle\alpha_{o s}\right\rangle=\right.$ $-1.50 \pm 0.10)$. Face-on accretion disks are brighter in the optical and would therefore be more abundant in our optically selected sample. However, it is not clear whether this selection effect is enough to explain the tight optical versus soft X-ray luminosity correlation found here.

If the soft $X$-ray flux originates in disk emission and the range of observed $\alpha_{x}$ is due to disk inclination effects, one would expect a steep $\alpha_{x}$ to be associated with a strong soft excess, rather than with a weak hard X-ray component, as observed here. Walter \& Fink (1993) have also concluded that disk models are inconsistent with their spectra, although they only use an ad hoc approximation for the expected accretion disk continuum shape and do not make detailed fits of their spectra. Note also, as mentioned above, that their distribution of $\alpha_{o x}$ and $\alpha_{o s}$ is likely to be strongly affected by their selection criteria, which affect the strength of their correlations. A quantitative comparison of the observed versus predicted correlations is required. This analysis will be presented when the complete sample is analyzed.

\subsection{The Continuum-Line Correlations \\ 5.3.1. Comparison with Previous Studies}

A remarkably strong correlation is suggested in our sample between the FWHM of $H \beta$ and $\alpha_{x}$, where $H \beta$ becomes narrower as $\alpha_{x}$ gets steeper ( $\S 4.2 .2$; Fig. 5 ). This correlation is consistent with the result of Puchnarewicz et al., who found that $\sim 50 \%$ of their USS AGNs have narrow $\mathrm{H} \beta$ (i.e., FWHM $\leq 2000 \mathrm{~km} \mathrm{~s}^{-1}$ ) versus only $23 \%$ in optically selected AGNs (as measured from Table 2 in Boroson \& Green 1992).

A significant correlation is also present between the $\mathrm{H} \beta$ FWHM and $\alpha_{o x}\left(r_{\mathrm{S}}=0.770, \operatorname{Pr}=0.0092\right)$, which is not surprising given the strong $\alpha_{o x}$ versus $\alpha_{x}$ correlation ( $\left.\$ 4.1\right)$ and the $\mathrm{H} \beta$ FWHM versus $\alpha_{x}$ correlation. Puchnarewicz et al. suggested evidence for a related $\alpha_{o x}$ versus H $\alpha$ FWHM correlation in their sample.

A significantly weaker correlation is present between the $\mathrm{H} \beta$ FWHM and $L_{2 \mathrm{kev}}\left(r_{\mathrm{S}}=0.631, \operatorname{Pr}=0.050\right)$. A similar correlation was suggested by Kriss et al. (1980), and by Stephens (1989), but was absent in the study of Puchnarewicz et al.

We also find here a significant correlation between $\alpha_{x}$ and the $\mathrm{Fe}$ II/H $\beta$ flux ratio. A related correlation, of $\alpha_{x}$ with the Fe II EW, was first suggested by Wilkes, Elvis, \& McHardy (1987), but was later disputed by Boroson (1989) and Zheng \& O'Brien (1990). The recent study by Shastri et al. (1993) suggested this correlation is significant in radio-quiet quasars only. In our sample the $\alpha_{x}$ versus Fe II EW correlation for radio-quiet quasars is formally not significant $\left(r_{s}=-0.643\right.$, $\mathrm{Pr}=0.115$ ). However, since only seven radio-quiet quasars are available in the current analysis, we can reliably identify here only a very strong correlation.

The fact that $\alpha_{x}$ correlates well with $L_{[\mathrm{O} \text { III] }}, \mathrm{Fe} \mathrm{II} / \mathbf{H} \beta$, and the $\mathrm{H} \beta \mathrm{FWHM}$, and not only with one of these parameters, is not surprising in view of the correlations known to exist among 
these three parameters, as clearly demonstrated by Zheng \& O'Brien and Boroson \& Green. The analysis of our complete sample of 23 quasars will allow us to estimate which of the correlations mentioned above is likely to be more fundamental. The Harder X-ray slope is known to correlate with the radioloudness (Wilkes \& Elvis 1987). We cannot test this correlation reliably here since our sample is optically selected and it includes only four radio-loud quasars, of which three are analyzed here. However, as noted in $\S 5.1$, we do find that the three radio-loud quasars have a flatter $\alpha_{x}$ compared with the seven radio-quiet quasars. This is similar to the trend found at harder X-rays. We note in passing that the harder X-ray slope in radio-loud quasars correlates with the radio core dominance (Shastri et al. 1993). The presence of this correlation cannot be reliably tested in our sample because of the small number of radio-loud quasars.

We find a remarkably strong correlation between $L_{\mathbf{H} \beta}$ and $L_{2 \mathrm{kev}}(\$ 4.2 .1)$. This correlation was already noted in the past by Kriss et al. (1980) and Blumenthal, Keel, \& Miller (1982), although a larger scatter is clearly apparent in their plots. The luminosities of three other lines presented here, [O III], $\mathrm{He}$ II, and Fe II, also correlate well with $L_{2 \mathrm{kev}}$ or $L_{0.3 \mathrm{kev}}$. In a preliminary report on a study of AGNs with $R O S A T$, Wamsteker et al. (1992) suggest that the luminosity of $C$ IV $\lambda 1549$ is also strongly correlated with the X-ray luminosity. Given the strong continuum-continuum correlations mentioned above, it is not clear whether the correlations of line luminosities with the X-ray luminosity are fundamental, or whether they are secondary to stronger correlations of the line luminosity with the continuum luminosity at other parts of the spectrum (e.g., the far-UV).

Corbin (1993) found significant correlations of $L_{2 \mathrm{keV}}$ with $\mathrm{Fe} \mathrm{II} / \mathrm{H} \beta\left(r_{\mathrm{S}}=-0.474\right)$, and of $L_{2 \mathrm{keV}}$ with the $\mathrm{H} \beta$ asymmetry as defined by Boroson \& Green $\left(r_{\mathrm{S}}=-0.471\right)$. We find here a very similar correlation coefficient for the $L_{2 \mathrm{kev}}$ versus $\mathrm{Fe} \mathrm{II} / \mathrm{H} \beta$ correlation $\left(r_{\mathrm{s}}=-0.512\right)$, but given the small size of our sample compared to the sample of Corbin (10 vs. 55 objects), our correlation is not significant $(\mathrm{Pr}=0.130)$. In contrast with Corbin, we find no evidence for a correlation between the $\mathrm{H} \beta$ asymmetry and $L_{2 \mathrm{kev}}\left(r_{\mathrm{s}}=-0.064\right)$. However, it is possible that our current sample is too small for the detection of the correlation.

\subsubsection{Spectral Slope Variability}

As shown in Figure 5, $\alpha_{x}$ appears to be strongly correlated with $L_{\text {[O II]. }}$. The presence of such a strong correlation, together with the fact that $L_{[\mathrm{OII}]}$ is very unlikely to vary in quasars on timescales shorter than a few years, indicates that $\alpha_{x}$ also does not vary significantly, i.e., by more than $\sim 10 \%$ (as deduced from the scatter in Fig. 5), on these timescales. As mentioned above ( $\$ 4.2 .2$ ), 3C 273 displays large variations in $\alpha_{x}$, and this object indeed falls significantly outside the relations suggested between $\alpha_{x}$ and $L_{\text {[O III }}, \mathrm{Fe}$ II/H $\beta$, or the H $\beta$ FWHM. The RASS observation of 3C 273, which gives $\alpha_{x}=-1.29$ instead of the -0.94 found here, puts it in significantly better agreement with the suggested correlations.

\subsubsection{The Soft X-Ray Opacity}

As noted in $\S 4.2 .2$, the correlations of $\alpha_{x}$ with the $\mathbf{H} \beta$ FWHM, $L_{[\mathrm{O} \text { III] }}$, and the $\mathrm{Fe} \mathrm{II} / \mathrm{H} \beta$ flux ratio are all significantly stronger when we assume $N_{\mathrm{H}}=N_{\mathrm{HI}}^{\mathrm{Gal}}$, rather than when we allow $N_{\mathrm{H}}$ to be a free parameter. This indicates that the $\mathrm{H}_{\mathrm{I}}$ column in the Galaxy, as measured in $21 \mathrm{~cm}$, is a good indicator of the Galactic soft X-ray opacity. The typical $N_{\mathrm{HI}}^{\mathrm{Gal}}$ in our sample is $\sim 1.5 \times 10^{20} \mathrm{~cm}^{-2}$, and at this column the Galaxy becomes optically thick only below $\sim 0.2 \mathrm{keV}$. The main opacity source at $0.2 \mathrm{keV}$ is $\mathrm{He} \mathrm{I}$ (Morrison \& McCammon 1983; Osterbrock 1989). Thus, the strong correlations present when $N_{\mathrm{H}}=N_{\mathrm{HI}}^{\mathrm{Gal}}$ indicate that the $\mathrm{H}$ I column at high Galactic latitudes is very well correlated with the $\mathrm{He}$ I column.

The root mean square of $\Delta N_{\mathrm{H}} \equiv N_{\mathrm{H}}-N_{\mathrm{HI}}^{\mathrm{Gal}}$ for our sample is $\left\langle\Delta^{2} N_{\mathrm{H}}\right\rangle=5.7 \times 10^{19} \mathrm{~cm}^{-2}$ (Table 3), with $\left\langle\Delta^{2} N_{\mathrm{H}}\right\rangle /$ $\left\langle N_{\mathrm{HI}}^{\mathrm{Gal}}\right\rangle=0.36$. This level of dispersion is clearly enough to have a strong effect on the correlations, as is evident from the comparison of the right- and left-hand panels of Figure 5. The dispersion in the correlation between $N_{\mathrm{HI}}^{\mathrm{Gal}}$ and $N_{\mathrm{HeI}}^{\mathrm{Gal}}$ must therefore be significantly smaller, to allow the smaller scatter in the correlations shown in the left-hand panel of Figure 5. Note that the Stark et al. $N_{\mathrm{HI}}^{\mathrm{Gal}}$ are accurate to about $10 \%$ (see Elvis et al. 1989). Thus, the error in the Galactic absorption correction, when the stark et al. values are used at $N_{\mathrm{HI}}^{\mathrm{Gal}} \gtrsim 5 \times 10^{20}$ $\mathrm{cm}^{-2}$, is large enough to practically erase the $\alpha_{x}$ correlations discussed here. Our selection criteria of $N_{\mathrm{HI}}^{\mathrm{Gal}}<1.9 \times 10^{20}$ $\mathrm{cm}^{-2}$ was therefore critical for being able to identify the $\alpha_{x}$ correlations.

Walter \& Fink (1993) have found $\left\langle\Delta N_{\mathrm{H}}\right\rangle=(-1.0 \pm 1.6)$ $\times 10^{19} \mathrm{~cm}^{-2}$ averaged over 50 of the 58 quasars in their sample, which also indicates that, on the average, quasars have no significant excess absorption or excess emission above the Galactic absorption. The fact that the centroid of their $\Delta N_{\mathrm{H}}$ distribution is so close to zero suggests that $N_{\mathrm{HI}}^{\mathrm{Gal}}$ gives an unbiased estimate of the Galactic soft X-ray opacity.

Recent far-UV observations by Kimble et al. (1993) below $500 \AA$ have shown that $N_{\mathrm{HI}}^{\mathrm{Gal}} / N_{\mathrm{HeI}}^{\mathrm{Gal}}=11.6 \pm 1$ within a distance of a few parsecs from the Sun. Here we can only address the dispersion in the ratio of $N_{\mathrm{HI}}^{\mathrm{Gal}}$ to $N_{\mathrm{HeI}}^{\mathrm{Gal}}$ for different lines of sight, and not the absolute value of the ratio. For example, a uniform low-ionization layer at high Galactic latitudes, in which $\mathrm{H}$ II is associated with $\mathrm{He} \mathrm{I}$, will result in an underestimate of the true soft X-ray opacity and therefore a systematic bias in the best-fit $\alpha_{x}$, which will not affect the strength of the correlations in Table 6. A more accurate upper limit on the possible dispersion in the ratio of $N_{\mathrm{HI}}^{\mathrm{Gal}}$ to $N_{\mathrm{HeI}}^{\mathrm{Gal}}$ will be evaluated when the complete sample is available.

The presence of the strong correlations for $N_{\mathrm{H}}=N_{\mathrm{HI}}^{\mathrm{Gal}}$ also suggests the lack of a significant column of $\mathrm{He}$ I at the quasar's rest frame. The absorption cross section of $\mathrm{He} I$ at $0.2 \mathrm{keV}$ is $1.68 \times 10^{-19} \mathrm{~cm}^{2}$ (Osterbrock 1989), and the lack of a significant intrinsic absorption at rest frame $0.2 \mathrm{keV}$ therefore implies $N_{\mathrm{He} \text { I }}<5.9 \times 10^{18} \mathrm{~cm}^{-2}$ at the quasar's redshift. A stronger limit on the rest frame $N_{\mathrm{He}}$ can be placed in the handful of high-redshift quasars, where the detection of continuum emission at $\lambda<504 \AA$ (e.g., Reimers et al. 1992) implies $N_{\mathrm{He} \mathrm{I}} \ll 1.3$ $\times 10^{17} \mathrm{~cm}^{-2}$. A strong limit on $N_{\mathrm{H} \text { I }}$ at the quasar's rest frame is already available for intermediate to high redshift quasars, where the lack of a significant Lyman limit absorption edge implies $N_{\mathrm{HI}} \ll 1.6 \times 10^{17} \mathrm{~cm}^{-2}$.

Note that the lack of a significant optical depth in either $\mathrm{H}_{\mathrm{I}}$ or $\mathrm{He} \mathrm{I}$ associated with quasars is not surprising, given the large ionizing flux produced by the central continuum source. This flux will highly ionize any diffuse gas with the host galaxy, assuming the gas density is not much above the density in the local diffuse interstellar medium.

\subsubsection{Absolute Luminosity Indicators}

Some of the correlations found here might prove useful as absolute luminosity indicators for quasars. The two strongest 
correlations we find between the absolute continuum or line luminosity and parameters which describe the continuum shape or the line shape are $\alpha_{\text {irx }}$ versus $L_{[0 \text { III] }}\left(r_{\mathrm{S}}=0.867\right.$, $\operatorname{Pr}=0.0025)$ and $\alpha_{x}$ versus $L_{[0 \mathrm{III}}\left(r_{\mathrm{S}}=0.830, \operatorname{Pr}=0.0029\right)$, where $\alpha_{\text {irx }}=\log \left(f_{17.685} / f_{14.25}\right) / 3.435$. Note that the last correlation is significantly stronger if objects with a known variable $\alpha_{x}$ (i.e., 3C 273), or objects not well fit by a power law (i.e., PG $1114+445)$, are excluded $(\$ 4.2 .2)$.

Baldwin (1977) suggested that the EW of C IV $\lambda 1549$ might be a useful absolute luminosity indicator. However, more recent studies (e.g., Francis et al. 1992) demonstrated a very large scatter in the $\mathrm{C}$ IV EW versus continuum luminosity correlation, which does not allow the $\mathrm{C}$ IV EW to be a reliable luminosity indicator.

Analysis of our complete $R O S A T$ sample will give us a more reliable estimate of the intrinsic scatter in the correlations suggested above. Multivariate analysis might allow us to reduce the scatter in the correlations and establish a reliable luminosity indicator. If an accurate indicator is found, then observations of higher redshift quasars can be used as useful probes for the value of the cosmological constant $q_{0}$, as suggested by Baldwin (1977).

\subsubsection{Other Possible Physical Implications}

The strong correlation suggested between $\alpha_{x}$ and the $\mathrm{H} \beta$ FWHM, $L_{[\mathrm{O} I \mathrm{II}]}$, and the $\mathrm{Fe} \mathrm{II} / \mathrm{H} \beta$ ratio are surprising in view of the apparent lack of any clear physical relation between these parameters. What do these correlations tell us? Given our current level of understanding of AGNs, no definite conclusions can be drawn. Some speculations are given below.

The fact that $H \beta$ becomes narrower as $\alpha_{x}$ gets steeper might be interpreted as an inclination effect. Puchnarewicz et al. suggested that the excess of narrow-line quasars in the USS sample can be explained if the broad-line region (BLR) clouds do not have an isotropic velocity distribution, but rather tend to move in a specific plane, while the soft X-ray component is produced in a thick accretion disk which is coplanar with the BLR. However, in this case, a steep $\alpha_{x}$ which is observed in a "face-on" quasar will be associated with a strong soft excess, rather than a weak hard X-ray component, as suggested in our spectra. The inclination effect scenario can hold if the hard X-ray component becomes weaker when optically selected quasars are viewed face on. Such an inclination dependence might be expected if the hard $\mathrm{X}$-rays are produced by Comptonization of accretion disk thermal emission by high-energy nonthermal electrons above the disk (Ghisellini et al. 1991), or if the hard X-rays are emitted from the surface of a thin accretion disk at very small radii.

Alternatively, the $\mathbf{H} \beta$ FWHM versus $\alpha_{x}$ correlation might imply the following: according to Netzer \& Laor (1993) the BLR is confined to the volume where the dust embedded in the line-emitting gas sublimes. The dust sublimes at a distance of $R_{\mathrm{BLR}} \simeq 0.2 L_{46}^{1 / 2}$ pc (see Laor \& Draine 1993), and therefore if dense cool gas is present at all distances from the center, then the distance of the BLR is determined uniquely by the continuum luminosity. If the width of the broad emission lines is related to the virial velocity, then FWHM $\propto M^{1 / 2} R_{\mathrm{BLR}}^{-1 / 2}$, where $M$ is the mass at the center of the quasar. Using the above expression for $R_{\mathrm{BLR}}$ we get FWHM $\propto M^{1 / 2} L^{-1 / 4}$. The absolute luminosity in our sample (at $3000 \AA$ ) is not significantly correlated with the $\mathrm{H} \beta$ FWHM, as might have been expected from the last relation if $M$ were constant in all AGNs. Thus the range in $\mathrm{H} \beta$ FWHM would reflect a range in $M$, and a narrow
$\mathrm{H} \boldsymbol{\beta}$ would imply a small central mass and therefore a high luminosity in Eddington units $\left(L_{\text {Eddington }}=1.25\right.$ $\times 10^{38}\left(M / M_{\odot}\right)$ ergs $\left.s^{-1}\right)$. The observed dependence of $\alpha_{x}$ on the $\mathrm{H} \beta$ width might therefore reflect a dependence of the X-ray production mechanism above $2 \mathrm{keV}$ on the central mass, or on the luminosity in Eddington units.

The large range in $\alpha_{x}$ found here suggests very different ionizing continua when extrapolated from $0.2 \mathrm{keV}$ to lower energies. The line emission is obviously dependent on the shape of the X-ray ionizing continuum (e.g., Kwan 1986), and one therefore expects some of the line ratios to correlate with $\alpha_{x}$. Such correlations might not be very strong since the ionizing flux is strongly dependent on the flux just shortward of $912 \AA$ and also on the unknown position of the far-UV spectral break ( 5 5.2). Apart from the shape of the ionizing flux, the line ratios also depend strongly on the density, column density, and the composition of the photoionized gas. A significant $\mathrm{Fe}$ II/ $/ \mathrm{H} \beta$ versus $\alpha_{x}$ correlation is suggested in the spectra presented here. $\mathrm{Fe}$ II is thought to arise mostly at large optical depths, in the partly ionized layers of the BLR clouds, and a significant contribution to $H \beta$ is also possible from this layer (e.g., Kwan \& Krolik 1981). The deep, partly ionized layer is heated mostly by hard X-rays, and thus a correlation of the EW of Fe II and $\mathrm{H} \beta$ with $\alpha_{o x}$ is expected. However, no significant correlation is found here for the Fe II EW with $\alpha_{o x}\left(r_{\mathrm{S}}=-0.333, \mathrm{Pr}=0.35\right)$, and a marginal correlation is suggested for $\mathrm{H} \beta\left(r_{\mathrm{s}}=0.661\right.$, $\mathrm{Pr}=0.038)$. The dependence of the $\mathrm{Fe} \mathrm{II} / \mathrm{H} \beta$ ratio on photoionization model parameters, in particular the shape of incident continuum, should be further explored in order to understand the physical implications of the $\mathrm{Fe} \mathrm{II} / \mathrm{H} \beta$ versus $\alpha_{x}$ correlation.

The strong $L_{[\mathrm{O} \text { III] }}$ versus $\alpha_{x}$ correlation is surprising. If the narrow-line clouds have very large column densities, as suggested by Netzer \& Laor (1993), then X-ray heating can affect the line emission from large optical depths. However, one would expect only a small contribution to [O III] from the deep layers where the dominant ionization state of $O$ is probably lower. The $L_{[\mathrm{OII}]}$ versus $\alpha_{x}$ correlation is likely to be induced by some other, presumably stronger, correlation of $\alpha_{x}$ with another parameter which controls $L_{[0 \text { III] }}$. A study of the effects of X-ray heating on the narrow-line emission appears warranted.

\section{SUMMARY}

We defined a complete sample of 23 optically selected quasars which includes all the PG quasars at $z \leq 0.400$ and $N_{\mathrm{HI}}^{\mathrm{Gal}}<1.9 \times 10^{20} \mathrm{~cm}^{-2}$. High-S/N spectra of all these quasars will be obtained by the ROSAT PSPC. The spectra of 10 quasars already available are presented here. The high quality of the ROSAT spectra allow us to determine the best-fitting $\alpha_{x}$ with about an order of magnitude higher precision compared with previously available X-ray spectra. We compared our results with earlier soft X-ray observations and looked for possible correlations of the X-ray properties with other emission parameters. Our major results are the following:

1. The spectra of nine of the 10 quasars are consistent, to within $\sim 30 \%$, with a single power-law model at $0.2-2 \mathrm{keV}$. In two of the nine quasars there are statistically significant deviations at a level of $\lesssim 30 \%$. There is no strong evidence for significant soft excess emission with respect to the best-fit power law. A small excess absorption might be present in two of the nine objects. The average soft $X$-ray spectral slope is 
$\left\langle\alpha_{x}\right\rangle=-1.50$, which is significantly steeper than the slope above $2 \mathrm{keV}$, and steeper than the average soft X-ray slope $\left(\left\langle\alpha_{x}\right\rangle=-1.0\right)$ found by the Einstein IPC.

2. The $0.3 \mathrm{keV}$ flux can be predicted to better than a factor of 2 once the $1.69 \mu \mathrm{m}$ flux is given. This, together with the known low variability of the near-IR emission, implies that the $\mathrm{X}$-ray variability power spectrum of quasars flattens between $f \sim 10^{-5}$ and $f \sim 10^{-8} \mathrm{~Hz}$.

3. A steep $\alpha_{x}$ is mostly associated with a weak hard X-ray component, relative to the near-IR and optical emission, rather than a strong soft excess, and the scatter in the normalized 0.3 $\mathrm{keV}$ flux is significantly smaller than the scatter in the normalized $2 \mathrm{keV}$ flux. This argues against either thin or thick accretion disks as the origin of the soft X-ray emission, unless the observed disk properties are restricted to a small range in parameter space.

4. Strong correlations are suggested between $\alpha_{x}$ and the $\mathrm{H} \beta$ FWHM, $L_{[\mathrm{O} \text { II] }}$, or the $\mathrm{Fe} \mathrm{II} / \mathrm{H} \beta$ flux ratio, when $N_{\mathrm{H}}=N_{\mathrm{HI}}^{\mathrm{Gal}}$ is assumed. These correlations imply the following:

(a) The quasar's environment is likely to be optically thin down to $\sim 0.2 \mathrm{keV}$, which implies $N_{\mathrm{He} \mathrm{I}}<5.9 \times 10^{18} \mathrm{~cm}^{-2}$, unless all our quasars have a similar intrinsic absorbing column.

(b) In most objects $\alpha_{x}$ does not vary by more than $\sim 10 \%$ on timescales shorter than a few years. (c) $\alpha_{x}$ might be a useful absolute luminosity indicator in quasars.

(d) $N_{\mathrm{HI}}^{\mathrm{GaI}}$ is well correlated with $N_{\mathrm{He} \mathrm{I}}^{\mathrm{Gal}}$ at high Galactic latitudes.

It is not clear what physical mechanisms are responsible for the strong correlations suggested here between $\alpha_{x}$ and the emission line parameters mentioned above. Further theoretical studies on the effects of the X-ray emission on the broad-and narrow-line emission might clarify the physical source of these correlations.

Analysis of the complete sample of 23 quasars will be carried out when all the spectra are available. This analysis will allow us to establish the soft X-ray properties of optically selected quasars, to test the strength and significance of the correlations suggested here, and to put quantitative constraints on soft $\mathrm{X}$-ray emission models for AGNs.

We thank the referee for a thorough review and some helpful comments. This work was supported in part by NASA grant NAG 5-2087, NAG 5-1618, NAG 5-30934, NAGW 2201 (LTSARP), and NASA contract NAS 8-20751. A. L. acknowledges support by NSF grant PHY 92-45317.

\section{REFERENCES}

Arnaud, K. A., et al. 1985, MNRAS, 217, 105

Bahcall, J. N., et al. 1993, ApJS, 87, 1

Baldwin, J. A. 1977, ApJ, 214, 679

Baldwin, J. A., Wampler, E. J., \& Gaskell, C. M. 1989, ApJ, 338, 630

Barvainis, R. 1993, ApJ, 412, 513

Berriman, G., Schmidt, G. D., West, S. C., \& Stockman, H. S. 1990, ApJS, 74 869

Bevington, P. R. 1969, Data Reduction and Error Analysis for the Physical Sciences (New York: McGraw-Hill)

Blumenthal, G. R., Keel, W., \& Miller, J. S. 1982, ApJ, 257, 499

Boroson, T. A. 1989, ApJ, 343, L9

Boroson, T. A., \& Green, R. F. 1992, ApJS, 80, 109

Brunner, H., Friedrich, P., Zimmermann, H.-U., \& Staubert, R. 1992, in X-ray Emission from Active Galactic Nuclei and the Cosmic X-Ray Background, ed. W. Brinkmann \& J. Trümper (Garching: MPI), 198

Canizares, C. R., \& White, J. L. 1989, ApJ, 339, 27

Carleton, N. P., Elvis, M., Fabbiano, G., Willner, S. P., Lawrence, A., \& Ward, M. 1987, ApJ, 318, 595

Comastri, A., Setti, G., Zamorani, G., Elvis, M., Giommi, P., Wilkes, B. J., \& McDowell, J. C. 1992, ApJ, 384, 62

Corbin, M. R. 1993, ApJ, 403, L9

Córdova, F. A., Kartje, J., Thompson, R. J., Mason, K. O., Puchnarewicz, E. M., \& Harnden, F. R. 1992, ApJS, 81, 661

Cutri, R. M., Wisniewski, W. Z., Rieke, G. H., \& Lebofski, M. J. 1985, ApJ, 296, 423

Czerny, B., \& Elvis, M. 1987, ApJ, 321, 305

Della Ceca, R., Palumbo, G. G. C., Persic, M., Boldt, E. A., De Zotti, G., \& Marshall, E. E. 1990, ApJS, 72, 471

Dickey, J. M., \& Lockman, F. J. 1990, ARA\&A, 28, 215

Elvis, M., Giommi, P., Wilkes, B. J., \& McDowell, J. 1991, ApJ, 378, 537

Elvis, M., Lockman, F. J., \& Wilkes, B. J. 1989, AJ, 97, 777

Elvis, M., Wilkes, B. J., McDowell, J. C., Green, R. F., Bechtold, J., Willner, S. P., Polomski, E., \& Cutri, R. 1994, ApJS, in press

Ferland, G. F., Korista, K. T., \& Peterson, B. M. 1990, ApJ, 363, L21

Fiore, F., Elvis, M., Mathur, S., Wilkes, B. J., \& McDowell, J. C. 1993, ApJ, 415,129

Fiore, F., Elvis, M., Siemiginowska, A., Wilkes, B. J., \& McDowell, J. C. 1994, ApJ, 431, 515

Francis, P. J., Hewett, P. C., Foltz, C. B., \& Chaffee, F. H. 1992, ApJ, 398, 476

Francis, P. J., Hewett, P. C., Foltz, C. B., Chaffee, F. H., Weymann, R. J., \& Morris, S. 1991, ApJ, 373, 465

Ghisellini, G., George, I. M., Fabian, A. C., \& Done, C. 1991, MNRAS, 248, 14

Hewitt, A., \& Burbidge, G. 1980, ApJS, 43, 57

Kellerman, K. I., Saramek, R., Schmidt, M., Shaffer, D. B., \& Green, R. 1989, AJ, 98, 1195

Kendall, M., \& Stuart, A. 1977, The Advanced Theory of Statistics, vol. 2 (4th ed.; New York: Macmillian)
Kimble, R. A., et al. 1993, ApJ, 404, 663

Kriss, G. A. Canizares, C. R., \& Ricker, G. R. 1980, ApJ, 242, 492

Kruper, J. S., Urry, C. M., \& Canizares, C. R. 1990, ApJS, 74, 347

Kwan, J. 1986, ApJ, 305, 679

Kwan, J., \& Krolik, J. H. 1981, ApJ, 250, 478

Lanzetta, K. M., Turnshek, D. A., \& Sandoval, J. 1993, ApJS, 84, 109

Laor, A. 1990, MNRAS, 246, 369

Laor, A., \& Draine, B. T. 1993 , ApJ, 402,441

Laor, A., \& Netzer, H. 1989, MNRAS, 238, 897

Lawrence, A., \& Elvis, M. 1982, ApJ, 256, 410

Lawrence, A., \& Papadakis, I. 1993, ApJ, 414, L85

Lawrence, A., Watson, M. G., Pounds, K. A., \& Elvis, M. 1987, Nature, 325, 694

Lawson, A. J., Turner, M. J. L., Williams, O. R., Stewart, G. C., \& Saxton, R. D. 1992, MNRAS, 259, 743

Madau, P. 1988, ApJ, 327, 116

Masnou, J.-L., Wilkes, B. J., Elvis, M., Arnaud, K. A., \& McDowell, J. C. 1992 A\&A, 253, 35

Mathur, S., Wilkes, B., Elvis, M., \& Fiore, F. 1994, ApJ, 434, 493

McHardy, I. 1990, in Proc. 23d ESLAB Symp., ed. J. Hunt \& B. Battrick (Paris: ESA), 1111

Miller, P., Rawlings, S., \& Saunders, R. 1993, MNRAS, 263, 425

Morrison, R., \& McCammon, D. 1983, ApJ, 270, 119

Mushotzky, R. F. 1984, Adv. Space Res., $3(10), 157$

Mushotzky, R. F., Done, C., \& Pounds, K. A. 1993, ARA\&A, 31, 717

Nandra, K., \& Pounds, K. A. 1992, Nature, 359, 215

Netzer, H., \& Laor, A. 1993, ApJ, 404, L51

Neugebauer, G., Green, R. F., Mathews, K., Schmidt, M., Soifer, B. T., \& Bennet, J. 1987, ApJS, 63, 615

Neugebauer, G., Soifer, B. T., Mathews, K., \& Elias, J. H. 1989, AJ, 97, 957

Osterbrock, D. E. 1989, Astrophysics of Gaseous Nebulae and Active Galactic

Nuclei (Mill Valley, CA: University Science Books)

Press, W. H., Flannery, B. P., Teukolsky, S. A., \& Vetterling, W. T. 1989, Numerical Recipes: The Art of Scientific Computing (Cambridge: Cambridge Univ. Press)

Puchnarewicz, E. M., Mason, K. O., Cordóva, F. A., Kartje, J., BranduardiRaymont, G., Mittaz, J. P. D., Murdin, P. G., \& Allington-Smith, J. 1992 MNRAS, 256, 589

Reimers, D., Vogel, S., Hagen, H.-J., Engels, D., Groote, D., Wamsteker, W., Clavel, J., \& Rosa, M. R. 1992, Nature, 360, 561

Sanders, D. B., Phinney, E. S., Neugebauer, G., Soifer, B. T., \& Mathews, K. 1989, ApJ, 347, 29

Savage, B. D., et al. 1993, ApJ, 413, 116

Saxton, R. D., Turner, M. J. L., Williams, O. R., Stewart, G. C., Ohashi, T., \& Kii, T. 1993, MNRAS, 262, 63

Schmidt, M., \& Green, R. F. 1983, ApJ, 269, 352

Schwartz, D. A., \& Tucker, W. H. 1988, ApJ, 332, 157 


\section{LAOR ET AL.}

Shastri, P., Wilkes, B. J., Elvis, M., \& McDowell, J. 1993, ApJ, 410, 29

Stark, A. A., Gammie, C. F., Wilson, R. W., Bally, J., Linke, R. A., Heiles, C., \& Hurwitz, M. 1992, ApJS, 79, 77

Staubert, R. 1992, in X-Ray Emission from Active Galactic Nuclei and the of Cosmic X-Ray Background, ed. W. Brinkmann \& J. Trümper (Garching $\operatorname{lil}_{I \rightarrow 1}$ MPI), 42

Staubert, R., Fink, H., Courvoisier, T. J.-L., Ulrich, M.-H., Brunner, H., Zimmermann, U., Kendziorra, E., \& Otterbein, K. 1992, in Testing the AGN Paradigm, ed. S. S. Holt, S. G. Nef, \& C. M. Urry (New York: AIP), 366

Stephens, S. A. 1989, AJ, 97, 10

Tananbaum H., Avni, Y., Green, R. F., Schmidt, M., \& Zamorani, G. 1986, ApJ, 305, 57

Thompson, R. J., Puchnarewicz, E. M., Córdova, F. A., \& Mason, K. O. 1994, ApJ, 420, 136

Trümper, J. 1983, Adv. Space Res. 2 (4) 241

Turner, T. J., George, I. M., \& Mushotzky, R. F. 1993a, ApJ, 412, 72 Turner, T. J., Nandra, K., George, I. M., Fabian, A. C., \& Pounds, K. A. 1993b,
ApJ, 419, 127
Turner, T. J., \& Pounds, K. A. 1989, MNRAS, 240, 833

Véron-Cetty, M. P., \& Véron, P. 1991, A Catalogue of Quasars and Active Nuclei (5th ed.; Munich: ESO)

Walter, R., \& Fink, H. H. 1993, A\&A, 274, 105

Wamsteker, W., et al. 1992, in X-Ray Emission from Active Galactic Nuclei and the Cosmic X-Ray Background, ed. W. Brinkmann \& J. Trümper (Garching: MPI), 155

Wilkes, B. J., \& Elvis, M. 1987, ApJ, 323, 243

Wilkes, B. J., Elvis, M., \& McHardy, I. 1987, ApJ, 321, L23

Wilkes, B. J., Tananbaum, H., Worrall, D. M., Avni, Y., Oey, M. S., \& Flanagan J 1994 ApJS 92,53

Williams, O. R., et al. 1992, ApJ, 389, 157

Zamorani, G., Giommi, P., Maccacaro, T., \& Tananbaum, H. 1984, ApJ, 278, 28

Zheng, W., \& O’Brien, P. T. 1990, ApJ, 353, 433 